\title{
Extension of smooth functions in infinite dimensions, I: unions of convex sets
}

\author{
by \\ C. J. Atkin (Wellington)
}

\begin{abstract}
Let $f$ be a smooth function defined on a finite union $U$ of open convex sets in a locally convex Lindelöf space $E$. If, for every $x \in U$, the restriction of $f$ to a suitable neighbourhood of $x$ admits a smooth extension to the whole of $E$, then the restriction of $f$ to a union of convex sets that is strictly smaller than $U$ also admits a smooth extension to the whole of $E$.
\end{abstract}

1. General remarks on extension problems. The guiding idea of this paper, which is intended as the first of a series, is to see what can be done to avoid the common and highly restrictive assumption that the model of a smooth manifold admits $\mathrm{C}^{\infty}$ (or $\mathrm{C}^{p}$, for some $p \geq 1$ ) partitions of unity. In this first paper I shall be exclusively concerned with the models themselves, and with some theorems which at first sight may seem too special to be of much consequence. In later papers I shall show that they are, in fact, the foundation of a substantial theory.

There has been much work done on the question of the existence or non-existence of partitions of unity of various degrees of differentiability (in the sense of Fréchet) in particular Banach spaces. Bonic and Frampton [1] showed that there are restrictions on their existence in many classical spaces, and pointed out some remarkable consequences of this fact. Toruńczyk [8] showed that $\mathrm{C}^{\infty}$ partitions of unity exist in Hilbert spaces of any dimension. A copious reference for the detailed and subtle theory of differentiable functions in Banach spaces, as it has developed since then, is the book of Deville, Godefroy, and Zizler [3]. (A perhaps more recent and startling result is given by Deville [2], who shows that, if a Banach space $E$ does not contain a copy of $c_{0}$, then the question of $\mathrm{C}^{\infty}$ partitions of unity for $E$ is, as it were, determined at the level of polynomials.) In all this work, however, some basic questions about Banach manifolds have remained unsolved.

2000 Mathematics Subject Classification: Primary 46T20. 
The ideal theory of Banach manifolds, if such a thing may be imagined, and if it were to generalize finite-dimensional constructions satisfactorily, would be valid for all, or at least for all separable, Banach spaces as models; it might, for instance, assert, irrespective of the existence of smooth partitions of unity, that every separable $\mathrm{C}^{\infty}$ Banach manifold is $\mathrm{C}^{\infty}$-diffeomorphic to a manifold of a certain standard kind, such as a closed submanifold of some multiple of the model or an open subset of the model. Individual peculiarities of the model should not unduly influence the basic theory, although they must inevitably have consequences elsewhere. The crucial difficulty in the way of such a theory is the absence, in general, of a suitably rich collection of smooth functions with which to perform familiar constructions. $\mathrm{C}^{\infty}$ partitions of unity, when they exist, enable one to imitate many finite-dimensional techniques, but their rarity is the reason why, for instance, Elworthy in [6] employs considerable effort to weaken the differentiability class he requires. As far as I know, even the question he raises in the introduction to [6], whether every smooth Banach manifold (in fact he mentions only the models $C$ and $\ell^{1}$, which one would expect to be the most refractory) admits a non-constant smooth function, has never been answered. In due course, although not in this paper, I shall give a positive answer to this question, at least for separable manifolds, and show that much more is true.

To establish results of such generality, hard analysis is unlikely to be appropriate. Indeed, the arguments I employ might be described as "combinatorical" - they prove that a positive solution to an extension problem in the small leads, by a complicated procedure of piecing-together, to a positive solution in the large. It will turn out in the end that such a technique is quite adequate for many purposes, and it has the advantage of requiring only very weak assumptions. Specifically, the model $E$ must be at least a Lindelöf locally convex space (that is, one of which any open cover has a countable subcover-see [7], 3.8, pp. 247 et seqq.; recall that any Lindelöf space is paracompact). Any separable metrizable locally convex space is Lindelöf, and so is the dual of any normed space in the weak* topology, since it is $\sigma$-compact. I shall also assume, for verbal and conceptual convenience, that $E$ is Hausdorff. The arguments could easily be modified to the non-Hausdorff case, which, in the natural situations where one is dealing with continuous functions, is a trivial corollary anyway. The notion of "smoothness" need not, however, be made precise, the only demands being that the class of "smooth" functions should satisfy certain conditions which obviously ought to hold, and do indeed hold, for most reasonable theories of differentiability, and for many other classes of functions and their generalizations as well.

In this first paper, I shall consider an extension problem for finite unions of convex sets in the model itself. If the model is paracompact and admits partitions of unity of the appropriate kind, which can be used to piece to- 
gether functions in the usual way, the results presented here are quite trivial and their limitation to convex sets is unnecessary. In general, however, even these apparently weak results have surprisingly strong consequences.

It seems preferable for clarity's sake not to present the construction at the outset in the greatest generality possible; it can be adapted in many ways, some of which will be studied later. I have, however, included some potentially useful modifications of the basic idea that require relatively little extra argument. The subset " $X$ ", which appears in 3.1, 4.2, 5.1 and elsewhere, is introduced with a view to classes of functions, such as Besov spaces, that may be defined on "thin" subsets of $\mathbb{R}^{n}$ (see, for instance, Triebel [9], [10]), and 4.6, 5.5 are motivated by the example of abstract Wiener manifolds, for which the coordinate transformations preserve a dense subspace of the dual $([4],[5])$. The countability restrictions on the model already appear several times as an essential hypothesis.

The simplest extension problem is perhaps the following.

Problem 1.1. Let $E, F$ be normed spaces, with $E$ separable, and let $f: U \rightarrow F$ be a $\mathrm{C}^{\infty}$ map defined on the neighbourhood $U$ of 0 in $E$. Does there exist a $\mathrm{C}^{\infty}$ map $g: E \rightarrow F$ which agrees with $f$ on a neighbourhood $V$ of 0 in $U$ ?

In this formulation " $\mathrm{C}^{\infty}$ " is understood in the Fréchet sense. A similar question might be asked about other classes of functions, such as $\mathrm{C}^{\infty}$ maps with compact derivatives at all points, or maps which are bounded or have uniformly bounded derivatives. For the above case, one has for instance the simple result

THEOREM 1.2. Let $E$ be a normed space in which there is a $\mathrm{C}^{\infty}$ mapping $h: E \rightarrow E$ that is the identity on some neighbourhood $W$ of the origin, and such that $h(E)$ is bounded. Let $F$ be any normed space. Then, if $U$ is a neighbourhood of 0 in $E$ and $f: U \rightarrow F$ is $\mathrm{C}^{\infty}$, there are a neighbourhood $V$ of 0 in $U$ and $a \mathrm{C}^{\infty}$ function $g: E \rightarrow F$ such that $g|V=f| V$.

Proof. Suppose that $h \mid B_{E}(0 ; \varepsilon)$ is the identity, where $B_{E}(0 ; \varepsilon)$ as usual denotes the ball of radius $\varepsilon$ about the origin in $E$, and that $h(E) \subseteq B_{E}(0 ; r)$. (Thus $r>\varepsilon$, of course). Choose $\kappa>0$ so that $B_{E}(0 ; \kappa r) \subseteq U$, and define

$$
(\forall x \in E) \quad g(x):=f\left(\kappa h\left(\kappa^{-1} x\right)\right) .
$$

Then take $V:=B_{E}(0 ; \kappa \varepsilon)$ and the result follows.

REMARK 1.3. The proof of 1.2 shows also that, in this case, the extension operator $f \mapsto g:=\mathcal{E} f$ is linear (with respect to pointwise operations), and that the image of $\mathcal{E} f$ is included in the image of $f$. If, therefore, $f$ is bounded with respect to the norm in $F$, so is $\mathcal{E} f$, and its bound is no greater than that of $f$. If the mapping $h$ has other properties - for instance if its $n$th derivative 
is bounded on $E$ for all $n \leq N$-then $\mathcal{E}$ may have associated properties, such as being bounded with respect to the $\mathrm{C}^{N}$ norm on the space of functions on $U$ for which all derivatives up to the $N$ th are bounded on $U$.

The existence of a function such as $h$ in many "classical" Banach spaces is easily established; the most immediate example is $C$, in which $h$ may simply be composition with a bounded $\mathrm{C}^{\infty}$ function $\mathbb{R} \rightarrow \mathbb{R}$ which is the identity on a neighbourhood of 0 . It is not much harder to construct a $\mathrm{C}^{\infty}$ function $h$ in the other "extreme" space, $\ell^{1}$. On the other hand, there are classes of functions other than $\mathrm{C}^{\infty}$, most obviously $\mathrm{C}^{\omega}$, for which the question analogous to 1.1 has, in general, a negative answer.

If one assumes in 1.1 that $\operatorname{cl}_{E}(V)$ is included in $U, f$ must behave well on the boundary of $V$; there can be no "local" obstruction to the extension, such as a failure of local boundedness in $E$. It seems, therefore, quite conceivable, although I think it unlikely, that the answer to 1.1 is positive in any normed space. It is perhaps more plausible, especially in non-separable spaces, that the class of all functions $\mathrm{C}^{\infty}$ in the sense of Fréchet may be either too large or too small to have satisfactory extension properties for all normed spaces $E$ and $F$, but that some other class contains all the mappings of practical interest and has better properties. Generally speaking, the questions of the form 1.1 for various choices of function class are all different.

I shall therefore develop a theory which, in essence, defines the question away; my functions will have a suitable extension property by definition. It will eventually appear that the results are much more satisfactory than one might expect.

Definition 1.4. Let $\mathcal{F}$ be a class of functions defined on the open sets of the topological space $X$. One may say that $\mathcal{F}$ is a class on $X . \mathcal{F}$ is said to be locally defined if

(i) if $f: U \rightarrow Y$ is a function defined on the open set $U$ such that, for any $x \in U$, there is a neighbourhood $V$ of $x$ in $U$ for which $f \mid V \in \mathcal{F}$, then necessarily $f \in \mathcal{F}$; and

(ii) if $U, V$ are open in $X, V \subseteq U$, and $f: U \rightarrow Y$ is in $\mathcal{F}$, then $f \mid V \in \mathcal{F}$.

Definition 1.5. A function $f: U \rightarrow Y$ in the class $\mathcal{F}$ on $X$ is $\mathcal{F}$ extensible, or extensible in $\mathcal{F}$, if, for any $x \in U$, there are an open neighbourhood $V$ of $x$ in $U$ and a function $g: X \rightarrow Y$ in $\mathcal{F}$ such that $g|V=f| V$. (If every function in $\mathcal{F}$ is $\mathcal{F}$-extensible, $\mathcal{F}$ itself may be described as extensible.)

Definition 1.6. A function $f$, defined on an open subset $U$ of the locally convex Hausdorff space $E$, is finitely determined if there are a continuous linear surjection $P: E \rightarrow \mathbb{R}^{k}$ (for some natural number $k$ ) and a function $\phi$ on the open subset $P(U)$ of $\mathbb{R}^{k}$ such that $f=\phi \circ P$. In effect, a finitely 
determined function on $U$ is one which may be expressed as a function of finitely many continuous linear functionals. $f$ will be described as $\mathrm{C}^{p}$ if and only if $\phi$ is $\mathrm{C}^{p}$. (This is consistent with any reasonable a priori definition.)

The finitely determined functions on $U$ with values in a vector space $F$ clearly form a vector space; if they take values in an algebra, they form an algebra. (It is also possible to restrict the linear surjections considered in the definition, and thus have a somewhat larger class of admissible functions. See 3.6.)

Definition 1.7. Let $X$ be a subset of the locally convex space $E$, and let $Y$ be a convex set in a vector space $F$. The class $\mathcal{F}$ on $X$ is $Y$-admissible if it contains at least one mapping $X \rightarrow Y$, and if, whenever $f, g: X \rightarrow Y$ are mappings in $\mathcal{F}$ and $\psi: W \rightarrow[0,1]$ is a finitely determined $\mathrm{C}^{\infty}$ function on an open subset $W$ of $E$ which includes $X$, then $h: X \rightarrow Y$ is also in $\mathcal{F}$, where $h$ is defined by

$$
(\forall x \in X) \quad h(x)=\psi(x) f(x)+(1-\psi(x)) g(x) .
$$

Lemma 1.8. Let the space $X$ be a subset of a locally convex Hausdorff space $E$, and let $\mathcal{F}$ be a $Y$-admissible class of mappings from the open sets of $X$ (in the subspace topology) to the convex subset $Y$ of the vector space $F$. Suppose that $A$ is an open subset of $E, B$ is a closed subset of $E$, and $B \subseteq A$. Then, for any $\mathcal{F}$-extensible $f: A \cap X \rightarrow Y$ in $\mathcal{F}$, and any closed straight-line segment $J:=[a, b]$ in $A$, there exists a convex open neighbourhood $W$ of 0 in $E$ such that $J+W \subseteq A$ and there is a mapping $g: X \rightarrow Y$ in $\mathcal{F}$ for which

$$
g|(J+W) \cap B \cap X=f|(J+W) \cap B \cap X .
$$

Proof. For each $x \in A \cap X$, there is a convex open neighbourhood $U_{x}$ of 0 in $E$ such that $x+U_{x} \subseteq A$, and there is a function $h_{x}: X \rightarrow Y$ in $\mathcal{F}$ such that

$$
h_{x}\left|\left(x+U_{x}\right) \cap X=f\right|\left(x+U_{x}\right) \cap X .
$$

Define $A_{1}:=\bigcup_{x \in B \cap X}\left(x+\frac{1}{2} U_{x}\right)$, an open subset of $A$ including $B \cap X$.

Suppose that $y \in E$. If $y \notin B$, there is a neighbourhood $V_{y}$ of 0 in $E$ such that $\left(y+V_{y}\right) \cap B=\emptyset$. Take $g_{y}: X \rightarrow Y$ in this case to be any mapping in $\mathcal{F}$ (there is one, by Definition 1.7). If, on the other hand, $y \in A_{1}$, then there exists some $x \in B \cap X$ such that $y \in x+\frac{1}{2} U_{x}$. Choose such an $x$, and let $V_{y}:=\frac{1}{2} U_{x}$; hence $y+V_{y} \subseteq x+U_{x}$. By the definition of $U_{x}$, there is a function $h_{x}: X \rightarrow Y$ which agrees with $f$ on $\left(x+U_{x}\right) \cap X$. Let $g_{y}: X \rightarrow Y$ be $h_{x}$. Since $E \backslash B$ and $A_{1}$ constitute an open cover of $E$, this defines, for every $y \in E$, a neighbourhood $V_{y}$ of 0 in $E$ and a function $g_{y}: X \rightarrow Y$ such that $g_{y}\left|\left(y+V_{y}\right) \cap B \cap X=f\right|\left(y+V_{y}\right) \cap B \cap X$. 
There will be no loss of generality in supposing that $J$ is a rectilinear segment of the form $[-c, c]$, since this may be arranged by translating $\frac{1}{2}(a+b)$ to 0 and also translating the class $\mathcal{F}$ in the obvious way, if necessary. By the compactness of $J$, there is an open symmetric convex neighbourhood $W$ of the origin in $E$ such that, for any $z \in J$, there is a $y \in J$ for which $z+3 W \subseteq y+V_{y}$. There are two cases.

If $W \cap \mathbb{R} c$ is unbounded, then $J \subseteq W$, and $J+W \subseteq 2 W \subseteq y+V_{y}$ for some $y \in J$. The conclusion of the lemma therefore follows on taking $g:=g_{y}$.

If $W \cap \mathbb{R} c$ is bounded, let $\kappa c$ be a frontier point of $W$, and take $\phi$ to be a support functional for $W$ at $\kappa c$, such that $1=\phi(\kappa c)>\phi(v)$ for all $v \in W$. (This is where the local convexity of $E$ is needed.) Then

$$
\phi(W)=\phi(W \cap \mathbb{R} c)=(-1,1) .
$$

Take $x_{1}, \ldots, x_{n} \in J$ so that $\left\{x_{i}+W: 1 \leq i \leq n\right\}$ covers $J$. For each $i$, there is, by the choice of $W$, a function $g_{i}: X \rightarrow Y$ in $\mathcal{F}$ which agrees with $f$ on $\left(x_{i}+3 W\right) \cap B \cap X$. Construct non-negative $\mathrm{C}^{\infty}$ functions $\psi_{i}$ on $\mathbb{R}$ so that

$$
\begin{aligned}
& \operatorname{supp} \psi_{i} \subseteq \phi\left(\left(x_{i}+W\right) \cap \mathbb{R} c\right)=\left(\phi\left(x_{i}\right)-1, \phi\left(x_{i}\right)+1\right) \quad \text { for each } i, \\
& \sum_{i=1}^{n} \psi_{i}(t)=1 \quad \text { for each } t \in[-1,1],
\end{aligned}
$$

and define $g: X \rightarrow Y$ by $g(x):=\sum_{i=1}^{n} \psi_{i}(\phi(x)) g_{i}(x)$. As $\mathcal{F}$ is admissible, $g \in \mathcal{F}$, by Definition 1.7 .

Suppose $x \in(J+W) \cap B \cap X$ and $\psi_{i}(\phi(x))>0$. Then there is some $y \in J$ such that $x \in y+W$, and therefore $|\phi(x)-\phi(y)|<1$. Since $\phi(x) \in\left(\phi\left(x_{i}\right)-1, \phi\left(x_{i}\right)+1\right)$ by $(2)$, it follows that $\left|\phi(y)-\phi\left(x_{i}\right)\right|<2$ and (by (1), as $y, x_{i} \in J$ ) that $y \in x_{i}+2 W$, whence $x \in x_{i}+3 W$. Consequently, $g_{i}(x)=f(x)$. This holds for each $i$ for which $\psi_{i}(\phi(x))>0$, and therefore, as required,

$$
g(x)=\sum_{i=1}^{n} \psi_{i}(\phi(x)) f(x)=f(x) .
$$

This proof is a prototype of the more complicated arguments which follow. It admits many variants, some of which will be mentioned later.

\section{Collections of convex sets}

Definition 2.1. Let $A, B$ be subsets of an abelian topological group $G$. I shall say that $A$ is strongly included in $B$, or $B$ strongly includes $A$, as subsets of $G$, and write $A \prec B$, if there exists a neighbourhood $U$ of the identity in $G$ such that $A+U \subseteq B$. 
The definition holds automatically if $A=\emptyset$, when the sum is also empty. It is an infinite-dimensional substitute for the relation which is familiar in finite dimensions, that $A$ should be a subset of a compact subset of $B$. Of course I shall take $G$ to be a locally convex Hausdorff space.

Lemma 2.2. Let $A$ be a convex set in the locally convex Hausdorff space $E$, and let $Q \prec A$. Then there exists a closed convex set $R$ such that $\operatorname{cl}_{E}(Q) \prec \operatorname{int}_{E}(R)$ and $R \prec A$. In particular, the closed convex envelope of $Q$ is strongly included in $A$.

Proof. Let $U$ be a convex open neighbourhood of 0 such that $Q+U \subseteq A$, and define

$$
R_{0}:=\left\{x \in E: x+\frac{1}{3} U \subseteq A\right\} .
$$

Then $R_{0}$ is convex, as is $R:=\mathrm{cl}_{E}\left(R_{0}\right)$, which is also strongly included in $A$, as $R+\frac{1}{4} U \subseteq A$, for instance. Furthermore, $Q+\frac{2}{3} U \subseteq R_{0}$, from which trivially

$$
\operatorname{cl}_{E}(Q) \subseteq Q+\frac{1}{3} U, \quad \operatorname{cl}_{E}(Q)+\frac{1}{3} U \subseteq R_{0},
$$

so that $\operatorname{cl}_{E}(Q) \prec \operatorname{cl}_{E}(Q)+\frac{1}{3} U \subseteq \operatorname{int}_{E}\left(R_{0}\right) \subseteq \operatorname{int}_{E}(R)$.

Proposition 2.3. Let $U$ be a convex open set in the normed space $E$, and let $C$ be a non-null convex subset of $U$ which is closed in $E$. Then there exists a convex set $V$ open in $E$ such that $C \subseteq V$ and $\operatorname{cl}_{E}(V) \subseteq U$.

Proof. By translation, one may assume that $0 \in C$. If $U=E$, there is nothing to prove; so suppose that $U \neq E$. For each $x \in U$, define

$$
r_{U}(x):=\sup \left\{\delta \in \mathbb{R}: B_{E}(x ; \delta) \subseteq U\right\},
$$

which is finite. Then $B_{E}\left(x ; r_{U}(x)\right) \subseteq U,\left|r_{U}(x)-r_{U}(y)\right| \leq\|x-y\|$, and $r_{U}(\alpha x+\beta y) \geq \alpha r_{U}(x)+\beta r_{U}(y)$ for $x, y \in U$ and $\alpha, \beta \in[0,1]$ such that $\alpha+\beta=1$. Now define

$$
V:=\bigcup_{x \in C} B_{E}\left(x ; \frac{1}{3} r_{U}(x)\right)
$$

It is apparent that $V$ is open in $E$.

Suppose $y_{1}, y_{2} \in V$ and $\alpha, \beta \geq 0$ and $\alpha+\beta=1$. Then there are points $x_{1}, x_{2} \in C$ such that $\left\|y_{i}-x_{i}\right\|<\frac{1}{3} r_{U}\left(x_{i}\right)$ for $i=1,2$. But, therefore,

$$
\begin{aligned}
\left\|\left(\alpha y_{1}+\beta y_{2}\right)-\left(\alpha x_{1}+\beta x_{2}\right)\right\| & \leq \alpha\left\|y_{1}-x_{1}\right\|+\beta\left\|y_{2}-x_{2}\right\| \\
& <\frac{1}{3} \alpha r_{U}\left(x_{1}\right)+\frac{1}{3} \beta r_{U}\left(x_{2}\right) \leq \frac{1}{3} r_{U}\left(\alpha x_{1}+\beta x_{2}\right),
\end{aligned}
$$

which, since $\alpha x_{1}+\beta x_{2} \in C$, shows that $V$ is convex.

Its closure in $E$ is included in $U$. For suppose $y_{n} \rightarrow y$, where each $y_{n} \in V$. For each $n$, there is $x_{n} \in C$ such that $y_{n} \in B_{E}\left(x_{n} ; \frac{1}{3} r_{U}\left(x_{n}\right)\right)$. If $\delta:=\frac{1}{2} \lim \sup _{n \rightarrow \infty} r_{U}\left(x_{n}\right)>0$, one may take a subsequence and renumber 
to ensure that $r_{U}\left(x_{n}\right) \geq \delta$ for all $n$. If $n$ is so large that $\left\|y_{n}-y\right\|<\frac{1}{3} \delta$, then

$$
\left\|y-x_{n}\right\| \leq\left\|y-y_{n}\right\|+\left\|y_{n}-x_{n}\right\|<\frac{1}{3} \delta+\frac{1}{3} r_{U}\left(x_{n}\right) \leq \frac{2}{3} r_{U}\left(x_{n}\right) .
$$

In view of the definition of $r_{U}$, this proves that $y \in U$ in this case. So $\mathrm{cl}_{E}(V) \subseteq U$ as required.

If, on the other hand, $r_{U}\left(x_{n}\right) \rightarrow 0$, then $\left\|x_{n}-y_{n}\right\| \rightarrow 0$, and so $x_{n} \rightarrow y$ as well. As $C$ is closed, this proves that $y \in C \subseteq U$ in this case.

Definition 2.4. Let $E$ be a normed space, and let $C, U$ be sets in $E$ such that $C \subseteq U$. Then $C$ is said to be boundedly strongly included in $U$ (which I write $C \preceq U$ ) if, for every bounded subset $Q$ of $E, C \cap Q \prec U$. (This concept is only of importance in $\S 5$.)

REMARK 2.5. The function $r_{U}$ defined at (3) can be used to express $\prec$ and $\preceq: C \prec U$ is equivalent to "inf $x \in C r_{U}(x)>0$ ", and, consequently, $C \preceq U$ is equivalent to "inf $\operatorname{incC\cap Q}_{U} r_{U}(x)>0$ for all bounded sets $Q$ ".

It appears that 2.3 cannot be directly generalized to locally convex spaces. The difficulty arises when $C$ has empty interior; otherwise, there is the simple argument 2.7 .

Lemma 2.6. Let $E$ be a normed space, and let $C$ and $U$ be convex sets such that $C \preceq U$. Then there exists a convex set $V$ open in $E$ such that $C \preceq V$ and $\operatorname{cl}_{E}(V) \preceq U$.

Proof. Follow the proof of 2.3. That $C \preceq V$ is clear, since, for any bounded $Q$, there exists $\varepsilon>0$ such that $r_{U}(x) \geq \varepsilon$ for all $x \in C \cap Q$, and therefore

$$
V \supseteq(C \cap Q)+B_{E}\left(0 ; \frac{1}{3} \varepsilon\right) .
$$

Now take some fixed $y \notin U$ (the case $U=E$ being trivial), and set $L:=\frac{1}{2}\|y\|$. If $x \in U, r_{U}(x) \leq 2 L+\|x\|$. If $K>0$ and $z \in \operatorname{cl}_{E}(V) \cap B_{E}(0 ; 2 K)$, there is a sequence $\left(z_{n}\right)$ in $V \cap B_{E}(0 ; 2 K)$ such that $z_{n} \rightarrow z$. By the definition of $V$, each $z_{n} \in x_{n}+B_{E}\left(0 ; \frac{1}{3} r_{U}\left(x_{n}\right)\right)$ for some $x_{n} \in C$, and

$$
2 K>\left\|z_{n}\right\|>\left\|x_{n}\right\|-\frac{1}{3} r_{U}\left(x_{n}\right) \geq\left\|x_{n}\right\|-\frac{1}{3}\left(2 L+\left\|x_{n}\right\|\right),
$$

so that $x_{n} \in C \cap B_{E}(0 ; 3 K+L)$. As $C \preceq U$, there exists a $\delta>0$ (depending only on $K)$ such that $r_{U}\left(x_{n}\right)>\delta$ for all $n$, and then, for large enough $n$,

$$
\begin{aligned}
z+B_{E}\left(0 ; \frac{1}{3} \delta\right) & \subseteq B_{E}\left(z_{n} ; \frac{2}{3} \delta\right) \subseteq B_{E}\left(x_{n} ; \frac{1}{3} r_{U}\left(x_{n}\right)+\frac{2}{3} \delta\right) \\
& \subseteq B_{E}\left(x_{n} ; r_{U}\left(x_{n}\right)\right) \subseteq U .
\end{aligned}
$$

As this holds for all $z \in \operatorname{cl}_{E}(V) \cap B_{E}(0 ; 2 K)$, it follows that $\operatorname{cl}_{E}(V) \preceq U$.

Lemma 2.7. Let $E$ be a locally convex space. Suppose $C$ and $U$ are convex sets such that $\operatorname{int}_{E}(C) \neq \emptyset, C$ is closed, $U$ is open, and $C \subseteq U$. Then there is a closed convex set $V$ such that $C \subseteq \operatorname{int}_{E} V$ and $V \subseteq U$. 
Proof. Let the Minkowski functionals of $C, U$ be $p_{C}, p_{U}$ respectively. Take $V:=\left\{x \in E: \frac{1}{2}\left(p_{C}(x)+p_{U}(x)\right) \leq 1\right\}$.

Lemma 2.8. Let $E$ be a normed space; suppose that $B \prec A$, where $B$ is bounded, and $A$ is convex and open, in $E$. Then there exists a bounded convex open set $A^{\prime}$ such that $B \prec A^{\prime} \subseteq A$.

Proof. Let $U$ be a bounded convex neighbourhood of 0 in $E$ such that $B+U \subseteq A$. Then take $A^{\prime}$ to be the convex cover of $B+U$.

Definition 2.9. Suppose $\mathcal{A}:=\left\{A_{1}, \ldots, A_{u}\right\}$ is a finite indexed class consisting of subsets of a set $X$. (The indices need not in principle be natural numbers, or such a sequence $\{1, \ldots, u\})$. By an analogy which will soon become apparent, I shall call it a complex in $X$. The members of a complex need not be distinct or non-empty. When $X$ is a locally convex space $E$, a second complex in $E, \mathcal{B}:=\left\{B_{1}, \ldots, B_{v}\right\}$, is described as strongly included in $\mathcal{A}$, which I write as $\mathcal{B} \prec \mathcal{A}$, if it is indexed by exactly the same indices as $\mathcal{A}$ and, for each index $i, B_{i} \prec A_{i}$ as subsets of $E$. In such case it is clear, since the index set is finite, that there exists some neighbourhood $V$ of the origin in $E$ such that $B_{i}+V \subseteq A_{i}$ for all indices $i$ simultaneously.

When $X$ is a normed space $E$, I say that the complex $\mathcal{B}$ is boundedly strongly included in the complex $\mathcal{A}, \mathcal{B} \preceq \mathcal{A}$, if $\mathcal{A}$ and $\mathcal{B}$ have the same index sets and, for each index $i, B_{i} \preceq A_{i}$; that is, for each bounded set $Q$ of $E$, there is a neighbourhood $V$ of 0 in $E$ such that, for each index $i$, $\left(B_{i} \cap Q\right)+V \subseteq A_{i}$.

Definition 2.10. Let $\mathcal{A}:=\left\{A_{1}, \ldots, A_{v}\right\}$ be a complex in the set $X$. For any $\Delta \subseteq\{1, \ldots, v\}$, let $A_{\Delta}:=\bigcap_{u \in \Delta} A_{u}$. Then I call $\Delta$ a simplex of $\mathcal{A}$ whenever $A_{\Delta} \neq \emptyset$. (In particular, this definition makes $\emptyset$ a simplex). The subsets $A_{u}$, for $u \in \Delta$, are called the vertices of the simplex $\Delta$. The star of a simplex $\Delta$, or of any subset $\Delta$ of the index set, is $A^{\Delta}:=\bigcup_{u \in \Delta} A_{u}$. The dimension of a simplex of $\mathcal{A}$ is its cardinality diminished by 1 ; the dimension of the complex $\mathcal{A}$ is the largest dimension of a simplex $\Delta$ of $\mathcal{A}$; a simplex is maximal if it is not a proper subset of any other simplex. The order of $\mathcal{A}$ is the number of distinct simplices whose dimension is the dimension of $\mathcal{A}$. (These names are obviously suggested by an imprecise analogy with the nerves of coverings.) The carrier of $\mathcal{A}$ is the set $A:=\bigcup_{u=1}^{v} A_{u}=A^{\{1, \ldots, v\}}$. Analogous notations are to apply to other complexes; thus the complex denoted by $\mathcal{B}$ gives $B_{\Delta}, B^{\Delta}, B$.

REMARK 2.11. If $\mathcal{A}, \mathcal{B}$ are complexes in a locally convex space $E$, and $\mathcal{B} \prec \mathcal{A}$, then $B^{\Delta} \prec A^{\Delta}$ and $B_{\Delta} \prec A_{\Delta}$ for each subset $\Delta$ of the index set. Indeed, there is a neighbourhood $V$ of the origin in $E$ such that $B^{\Delta}+V \subseteq A^{\Delta}$ and $B_{\Delta}+V \subseteq A_{\Delta}$ for all $\Delta$. 
The main result of this paper will be Theorem 5.1, which says approximately that, if a complex $\mathcal{B}$ is strongly included in a complex $\mathcal{A}$ of convex open sets in a locally convex Hausdorff space $E$, and if $\mathcal{F}$ is an admissible class, then, for any $\mathcal{F}$-extensible function $f$ defined on the carrier of $\mathcal{A}$, there is a function in $\mathcal{F}$ defined on $E$ and agreeing with $f$ on the carrier of $\mathcal{B}$. The difficulty with the proof is that the complexes may have a complicated combinatorical structure, that is, the convex sets may intersect in a way that makes local extensions interfere with each other. There are therefore two steps; in the first I consider what happens if the simplest case, that of stars, is already known.

\section{Extension (stars of simplices excepted)}

Proposition 3.1. Let $E$ be a locally convex space, and $F$ a vector space, with $X$ a subset of $E$ and $Y$ a convex subset of $F$. Let $\mathcal{F}$ be a $Y$-admissible class (see 1.7) of functions defined on the open subsets of $X$ with values in $Y$. Suppose that $\mathcal{A}:=\left\{A_{u}: 1 \leq u \leq v\right\}$ is a complex consisting of open convex sets of $E$, and $\mathcal{B}$ is a complex of subsets of $E$, such that $\mathcal{B} \prec \mathcal{A}$. Then, for any map $f: B \cap X \rightarrow Y$, and any family of maps $f_{\Delta}: X \rightarrow Y$ in $\mathcal{F}$, one for each simplex $\Delta$ of $\mathcal{A}$, such that $f_{\Delta}\left|B^{\Delta} \cap X=f\right| B^{\Delta} \cap X$ for each $\Delta$, there exists a map $h: X \rightarrow Y$ in $\mathcal{F}$ such that $h|B \cap X=f| B \cap X$.

Remark 3.2. Every simplex of $\mathcal{B}$ is a simplex of $\mathcal{A}$, but the converse is not true; that is, there may be many simplices $\Delta$ of $\mathcal{A}\left(A_{\Delta} \neq \emptyset\right)$ for which $B_{\Delta}=\emptyset$. It is also quite possible that $B^{\Delta} \cap X=\emptyset$, in which case the condition on $f_{\Delta}$ would be vacuously satisfied. The reason for introducing $X$ is (see $\S 1$ ) that there are some interesting classes of functions which can only be reasonably defined on "thin" sets. See 3.3 for further comments.

Proof of 3.1. The argument is inductive, and will be presented in steps.

(a) If the dimension of $\mathcal{A}$ is -1 , all its members and all the members of $\mathcal{B}$ are null; and, whenever $B=\emptyset$, the proposition holds trivially because, by 1.7 , there is some map $h: X \rightarrow Y$ in $\mathcal{F}$.

(b) If the dimension of $\mathcal{A}$ is greater than -1, there are some 0 -simplices in $\mathcal{A}$, that is, indices $u$ for which $A_{u} \neq \emptyset$. Clearly, any other indices may simply be ignored, so I shall assume henceforth that $A_{u}$ is non-null for each $u$. Of course, some of the $B_{u}$ may be null.

(c) If the dimension of $\mathcal{A}$ is $v-1$, every 0 -simplex is a vertex of a unique simplex $\Delta$ of maximal dimension; the order of $\mathcal{A}$ must be 1 , and $A=A^{\Delta}$. The proposition is trivially true, for it suffices to take $h:=f_{\Delta}$. In particular, this applies if there is a single vertex $(v=1, d=0, o=1)$.

(d) Suppose now that the dimension of $\mathcal{A}$ is $d \geq 0$ and the order of $\mathcal{A}$ is $o$. The inductive hypothesis will be that the proposition has already been 
proved for any $\mathcal{A}$ and $\mathcal{B}$ satisfying the hypotheses, provided that $\mathcal{A}$ has dimension less than $d$, or, when $o \geq 2$, dimension equal to $d$ and order less than $o$. The cases $d=-1$ and $d=0, o=1$ have been proved at (a) and (c).

(e) (c) also applies when $d+1=v$. So now, suppose $d+1<v$. Renumbering, if necessary, I may suppose that

$$
Q:=A_{1} \cap \ldots \cap A_{d+1} \neq \emptyset
$$

is a simplex of the maximum dimension $d$. For each $u$ with $d+1<u \leq v$, $Q \cap A_{u}=\emptyset$ (or there would be a simplex of dimension $d+1$ ). As $Q$ and $A_{u}$ are disjoint open non-empty convex sets, one has for each such $u$ a continuous non-zero linear functional $\lambda_{u}$ on $E$, and a scalar $\varrho_{u}$, such that

$$
\lambda_{u}(Q) \subseteq\left\{\tau \in \mathbb{R}: \tau<\varrho_{u}\right\}, \quad \lambda_{u}\left(A_{u}\right) \subseteq\left\{\tau \in \mathbb{R}: \tau>\varrho_{u}\right\} .
$$

The functionals $\lambda_{d+2}, \lambda_{d+3}, \ldots, \lambda_{v} \in E^{\prime}$ need not be linearly independent. Choose from them a maximal linearly independent subset, which, by renumbering, may be denoted as $\left\{\lambda_{v-k+1}, \ldots, \lambda_{v}\right\}$. Define

$$
P: E \rightarrow \mathbb{R}^{k}: x \mapsto\left(\lambda_{v-k+1}(x), \ldots, \lambda_{v}(x)\right) .
$$

Then $P$ is a continuous epimorphism. Each of the functionals $\lambda_{u}, \lambda_{u+1}, \ldots$ $\ldots, \lambda_{v}$ for $d+1<u \leq v$ is of the form $\mu_{u} \circ P$ for some suitable non-zero linear functional $\mu_{u}$ on $\mathbb{R}^{k}$. Hence, from (4),

$$
\left\{\begin{array}{l}
P(Q) \subseteq\left\{\xi \in \mathbb{R}^{k}:(\forall u) d+1<u \leq v \Rightarrow \mu_{u}(\xi)<\varrho_{u}\right\} \\
P\left(A_{u}\right) \subseteq\left\{\xi \in \mathbb{R}^{k}: \mu_{u}(\xi)>\varrho_{u}\right\} \text { for } d+1<u \leq v
\end{array}\right.
$$

Now $B_{u} \prec A_{u}$ for each $u$; there exists a convex neighbourhood $V$ of 0 in $E$ such that, for $1 \leq u \leq v, B_{u}+3 V \subseteq A_{u}$. For each $u$ define

$$
\left\{\begin{array}{l}
A_{u}^{\prime}:=\left\{x \in E: x+V \prec A_{u}\right\}, \\
Q^{\prime}:=A_{1}^{\prime} \cap \ldots \cap A_{d+1}^{\prime} ; \quad \text { thus } \\
B_{u}+V \subseteq A_{u}^{\prime}, \quad A_{u}^{\prime}+V \subseteq A_{u},
\end{array}\right.
$$

so that $B_{u} \prec A_{u}^{\prime} \prec A_{u}$, and $A_{u}^{\prime}$ is an open convex set. Also, $Q^{\prime}+V \subseteq Q$. It is of course possible that $Q^{\prime}=\emptyset$. Since the functionals $\lambda_{u}=\mu_{u} \circ P$ are non-zero, there exists $\varepsilon>0$ such that $\mu_{u}(P(V)) \supseteq(-2 \varepsilon, 2 \varepsilon)$ for every $u$. Consequently, (6) gives

$$
\left\{\begin{array}{l}
P\left(Q^{\prime}\right) \subseteq\left\{\xi \in \mathbb{R}^{k}:(\forall u) d+1<u \leq v \Rightarrow \mu_{u}(\xi)<\varrho_{u}-2 \varepsilon\right\}, \\
P\left(A_{u}^{\prime}\right) \subseteq\left\{\xi \in \mathbb{R}^{k}: \mu_{u}(\xi)>\varrho_{u}+2 \varepsilon\right\} \quad \text { for } d+1<u \leq v
\end{array}\right.
$$

For each subset $T$ of $\{d+2, d+3, \ldots, v\}$, set

$$
\left\{\begin{array}{c}
T^{\mathrm{c}}:=\{d+2, d+3, \ldots, v\} \backslash T, \\
U(T):=\left\{\xi \in \mathbb{R}^{k}:(\forall r \in T)\left(\mu_{r}(\xi)>\varrho_{r}-2 \varepsilon\right)\right. \\
\left.\quad \&\left(\forall r \in T^{\mathrm{c}}\right)\left(\mu_{r}(\xi)<\varrho_{r}+2 \varepsilon\right)\right\}, \\
C(T):=\left\{\xi \in \mathbb{R}^{k}:(\forall r \in T) \quad\left(\mu_{r}(\xi) \geq \varrho_{r}-\varepsilon\right)\right. \\
\left.\quad \&\left(\forall r \in T^{\mathrm{c}}\right)\left(\mu_{r}(\xi) \leq \varrho_{r}+\varepsilon\right)\right\} .
\end{array}\right.
$$


The sets $C(T)$ are closed in $\mathbb{R}^{k} ; U(T)$ is open and convex, $C(T) \prec U(T)$. Define, for each $T$ and for $1 \leq u \leq v$,

$$
A_{u}(T):=A_{u}^{\prime} \cap P^{-1}(U(T)), \quad B_{u}(T):=B_{u} \cap P^{-1}(C(T)) .
$$

Thus the sets $A_{u}(T)$ are open and convex in $E$.

There is a convex neighbourhood $W$ of 0 in $E$ such that $W \subseteq V$ and, for each $u, \lambda_{u}(W) \subseteq(-\varepsilon, \varepsilon)$. If $x \in B_{u}(T)$, then (see (7)) $x+V \subseteq A_{u}^{\prime}$. Also, for all $r>d+1, P(x) \in C(T)$ and

$$
\lambda_{r}(x+W) \subseteq\left(\lambda_{r}(x)-\varepsilon, \lambda_{r}(x)+\varepsilon\right) ;
$$

for $r \in T$, therefore, (9) shows $\left(\mu_{r} \circ P\right)(x+W) \subseteq\left(\varrho_{r}-2 \varepsilon, \infty\right)$, whilst, when $r \in T^{\mathrm{c}},\left(\mu_{r} \circ P\right)(x+W) \subseteq\left(-\infty, \varrho_{r}+2 \varepsilon\right)$. So $P\left(B_{u}(T)+W\right) \subseteq U(T)$; hence, $B_{u}(T)+W \subseteq A_{u}(T)$, and

$$
\mathcal{B}(T):=\left\{B_{1}(T), \ldots, B_{v}(T)\right\} \prec \mathcal{A}(T):=\left\{A_{1}(T), \ldots, A_{v}(T)\right\} .
$$

Each set of $\mathcal{A}(T)$ or of $\mathcal{B}(T)$ is a subset of the corresponding set of $\mathcal{A}$ or of $\mathcal{B}$, and each simplex of $\mathcal{A}(T)$ is a simplex of $\mathcal{A}$. Consequently, the hypotheses of the proposition are satisfied for the pair $\mathcal{A}(T), \mathcal{B}(T)$, if one takes instead of $f$ its restriction to $B(T) \cap X$ and the same $f_{\Delta}$ as before for every simplex $\Delta$ of $\mathcal{A}(T)$.

(f) From (10) and (8), one sees that, for $d+1<u \leq v$,

$$
\lambda_{u}\left(A_{u}(\emptyset)\right) \subseteq \lambda_{u}\left(A_{u}^{\prime}\right) \subseteq\left(\varrho_{u}+2 \varepsilon, \infty\right) .
$$

But (9) and (10) ensure that $\lambda_{u}\left(A_{u}(\emptyset)\right) \subseteq \mu_{u}(U(\emptyset)) \subseteq\left(-\infty, \varrho_{u}+2 \varepsilon\right)$ for $d+1<u \leq v$. So $A_{u}(\emptyset)=\emptyset$ for $d+1<u \leq v$, and the only 0 -simplices (non-null members) of $\mathcal{A}(\emptyset)$ must be among $A_{1}(\emptyset), \ldots, A_{d+1}(\emptyset)$. Therefore either the dimension of $\mathcal{A}(\emptyset)$ is $d$, and its order is 1 , or its dimension is less than $d$. The first case was discussed at (c). In the second case, the inductive hypothesis (d) applies to the pair $\mathcal{A}(\emptyset), \mathcal{B}(\emptyset)$. In both cases, there is a function $h_{\emptyset}: X \rightarrow Y$ such that $h_{\emptyset}|B(\emptyset) \cap X=f| B(\emptyset) \cap X$.

(g) If $T \neq \emptyset$, let $r \in T$. Then, for all $u, 1 \leq u \leq v$, (9) and (10) entail

$$
\lambda_{r}\left(A_{u}(T)\right) \subseteq \mu_{r}(U(T)) \subseteq\left(\varrho_{r}-2 \varepsilon, \infty\right) .
$$

On the other hand, (10) and (8) show that, for $d+1<r \leq v$,

$$
\lambda_{r}\left(\bigcap_{u=1}^{d+1} A_{u}(T)\right) \subseteq \lambda_{r}\left(\bigcap_{u=1}^{d+1} A_{u}^{\prime}\right)=\lambda_{r}\left(Q^{\prime}\right) \subseteq\left(-\infty, \varrho_{r}-2 \varepsilon\right) .
$$

By (11) and (12), necessarily $A_{1}(T) \cap \ldots \cap A_{d+1}(T)=\emptyset$. Now every simplex of $\mathcal{A}(T)$ is a simplex of $\mathcal{A}$, and at least one $d$-simplex of $\mathcal{A}$ is not a simplex of $\mathcal{A}(T)$; hence either the dimension of $\mathcal{A}(T)$ is less than $d$ or its order is less than $o$. In either case, the inductive hypothesis (d) applies to the pair $\mathcal{A}(T), \mathcal{B}(T)$. 
(h) For all choices of $T$, then, there is a function $h_{T}: X \rightarrow Y$ such that $h_{T}|B(T) \cap X=f| B(T) \cap X$. Note from (10) that

$$
B(T)=B \cap P^{-1}(C(T)) .
$$

From (9), any $\xi \in \mathbb{R}^{k}$ lies in the interior of $C\left(T_{\xi}\right)$, if $T_{\xi}$ denotes the set of indices $r \in\{d+2, d+3, \ldots, v\}$ for which $\mu_{r}(\xi) \geq \varrho_{r}$. So the interiors of the sets $C(T)$ cover $\mathbb{R}^{k}$. There is a subordinate $\mathrm{C}^{\infty}$ partition of unity $\left\{\psi_{T}\right\}$ on $\mathbb{R}^{k}$, where $C(T)$ includes the support of $\psi_{T}$ for each $T$. Certainly, $\psi_{T} \circ P$ is finitely determined (see 1.6). Thus $h: X \rightarrow Y$, defined by

$$
h(x):=\sum_{T} \psi_{T}(P(x)) h_{T}(x),
$$

where the sum extends over all $2^{v-d-1}$ choices of $T \subseteq\{d+2, d+3, \ldots, v\}$, is in $\mathcal{F}$ by 1.7 , and it takes values in $Y$ because $Y$ is convex. Suppose $x \in B \cap X$. For any $T$ such that $\psi_{T}(P(x)) \neq 0$, then, $P(x) \in C(T)$ and, from (10), $x \in B(T) \cap X$. Ergo, $h_{T}(x)=f(x)$ by the construction. So $h|B \cap X=f| B \cap X$ as required. This completes the inductive step, in the only case $(d+1<v)$ for which it was non-trivial. The proof is therefore finished.

REMARK 3.3. In the argument above, there is no need to assume any countability hypothesis for $E$, or to demand that the class $\mathcal{F}$ be locally defined, because the construction of $h$ terminates after finitely many steps. This is no longer true in $\S 4$. Also, notice that the only extensions of $f$ that are needed are explicitly demanded in the conditions of the theorem, and, consequently, that $f$ is automatically $\mathcal{F}$-extensible.

As is evident in the proof, the rôle of the complex $\mathcal{A}$ in the statement of the theorem is merely to furnish a convenient class of simplices to which the conditions that have to be imposed may apply; it does not appear in the conclusion. It would be more natural to begin with the complex consisting of the convex envelopes $\Gamma(B)$ of the elements $B$ of $\mathcal{B}$, but, no matter how small a neighbourhood $V$ of 0 were taken, the complex $\{\Gamma(B)+V: B \in \mathcal{B}\}$ might have additional simplices.

The underlying idea is that linear functionals and smooth combinations of them give a large enough class of $\mathrm{C}^{\infty}$ functions for some purposes. Specifically, the Hahn-Banach theorem enables one to use them to separate combinations of open convex sets. It might theoretically be possible to argue similarly on the basis of other separation theorems, which might be a way to improve the results and even to extend them to non-separable spaces; but I do not know of any such theorems. For instance, can two disjoint closed polynomially-convex sets always be separated by a smooth function? As things are, the requirements that $\mathcal{A}$ be finite and consist of convex sets 
force the later discussion to be limited to spaces with some countability restriction; if, for instance, $\mathcal{A}$ could be locally finite but uncountable, more might be proved.

There are several possible addenda to Proposition 3.1, two of which may be given here. It is convenient to establish the notation $\mathcal{F}(M ; N)$ to mean the set of functions $M \rightarrow N$ that belong to $\mathcal{F}$. Any algebraic operations in $\mathcal{F}(M ; N)$ are understood to be defined pointwise when they are defined. The proof of 3.1 yields, without serious alteration:

Corollary 3.4. Let $\mathcal{A}, \mathcal{B}$ and $\mathcal{F}, E, F, X$ be as in 3.1. Suppose that $\mathcal{E}$ is a convex set of functions $A \cap X \rightarrow F$ and that, for each simplex $\Delta$ of $\mathcal{A}$ and for each $f \in \mathcal{E}$, there is an affine-linear mapping $\mathcal{L}_{\Delta}: \mathcal{E} \rightarrow \mathcal{F}(X ; F)$ such that $\mathcal{L}_{\Delta}(f)\left|B^{\Delta} \cap X=f\right| B^{\Delta} \cap X$. Then there is an affine-linear map $\mathcal{L}: \mathcal{E} \rightarrow \mathcal{F}(X ; F)$ such that, for each $f \in \mathcal{E}, \mathcal{L}(f)|B \cap X=f| B \cap X$. Furthermore, the image of $\mathcal{L}(f)$ is included in the convex envelope of all the images of the mappings $\mathcal{L}_{\Delta}(f)$.

REMARK 3.5. The corollary arises from the observation that all the constructions in the proof of 3.1-the linear functionals $\lambda_{u}$, the pairs $\mathcal{A}(T), \mathcal{B}(T)$, and the partition of unity $\psi_{T}$-are made to accord only with the geometry of $\mathcal{A}$ and $\mathcal{B}$, and do not depend on the functions $f, f_{\Delta}$.

Definition 3.6. Suppose that $G$ is a subspace of the topological dual $E^{\prime}$ of the locally convex Hausdorff space $E$. A linear surjection $P: E \rightarrow \mathbb{R}^{k}$ will be called a $G$-surjection if its composition with each of the coordinate functions of $\mathbb{R}^{k}$ is a linear functional belonging to $G$. (Its composition with any linear functional on $\mathbb{R}^{k}$ then also belongs to $G$.) A function $f: U \rightarrow \mathbb{R}$ defined on an open set $U$ of $E$ may be described as $G$-finitely determined if there exist a $G$-surjection $P: E \rightarrow \mathbb{R}^{k}$ and a function $\phi: P(U) \rightarrow \mathbb{R}$ such that $f=\phi \circ P$. If $X$ is a subset of $E$ and $Y$ is a convex set in the vector space $F$, a class $\mathcal{F}$ of functions $X \rightarrow Y$ is $(G, Y)$-admissible if, whenever $f, g$ : $X \rightarrow Y$ are mappings in $\mathcal{F}$ and $\psi: W \rightarrow[0,1]$ is a $G$-finitely determined $\mathrm{C}^{\infty}$ function on an open subset $W$ of $E$ which includes $X$, then $h: X \rightarrow Y$ is also in $\mathcal{F}$, where

$$
(\forall x \in X) \quad h(x)=\psi(x) f(x)+(1-\psi(x)) g(x) .
$$

This is, of course, a less demanding condition, in principle, than the $Y$-admissibility of 1.7 .

Lemma 3.7. Suppose in 3.1 or in 3.4 that $E$ is a normed space and all the convex sets belonging to the complex $\mathcal{A}$ are bounded. Let $G$ be a norm-dense subspace of the dual space $E^{\prime}$. Then the previous conclusions, in 3.1 or in 3.4 , hold on the weaker condition that $\mathcal{F}$ is $(G, Y)$-admissible instead of $Y$-admissible. 
Proof. Suppose that all the sets of $\mathcal{A}$ lie in $B_{E}(0 ; L)$. Define the functionals $\lambda_{u}$ as at (4), and the sets $A_{u}^{\prime}, Q^{\prime}$ as at (7). Then there exists an $\varepsilon>0$ such that, for each $u$ such that $d+1<u \leq v, \sup \lambda_{u}\left(Q^{\prime}\right)+6 \varepsilon<\inf \lambda_{u}\left(A_{u}^{\prime}\right)$. If $\lambda_{u}^{\prime} \in E^{\prime}$ and $\left\|\lambda_{u}-\lambda_{u}^{\prime}\right\|<\varepsilon / L$, then $\sup \lambda_{u}^{\prime}\left(Q^{\prime}\right)<\sup \lambda_{u}\left(Q^{\prime}\right)+\varepsilon$ and $\inf \lambda_{u}^{\prime}\left(Q^{\prime}\right)>\sup \lambda_{u}^{\prime}\left(Q^{\prime}\right)-\varepsilon$ for each $u$ with $d+1<u \leq v$. Hence, one may choose elements $\lambda_{u}^{\prime}$ of $G$ such that $\sup \lambda_{u}^{\prime}\left(Q^{\prime}\right)+4 \varepsilon<\inf \lambda_{u}^{\prime}\left(A_{u}^{\prime}\right)$. Select a maximal linearly independent subset (for infinite-dimensional $E$, the $\lambda_{u}^{\prime}$ might already have been chosen to be linearly independent), and proceed as before; (8) becomes a consequence of the new construction.

REMARK 3.8. What was needed at 3.1(e) was a condition on two disjoint convex sets $Q, R$ that would ensure the existence of $\lambda \in E^{\prime}$ such that the intervals $\lambda(Q), \lambda(R)$ of $\mathbb{R}$ are "separated" - in effect, have disjoint closures. The condition used was that $(Q+V) \cap R=\emptyset$ for some neighbourhood $V$ of 0 in $E$, which one might call convex separation; then 3.7 says that, if $Q$ and $R$ are non-null separated convex sets and bounded in the normed space $E$, the set of possible $\lambda$ is norm-open in $E^{\prime}$. If $Q$ is unbounded, there is often only one possible $\lambda$, whilst, if $Q$ and $R$ are disjoint non-null, convex, bounded and closed, but not separated, the set of separating linear functionals is not norm-open.

4. Extension from stars of simplices. The question of existence of the extension $h$ is reduced by 3.1 to the existence of the functions $f_{\Delta}$, the "extensions from stars" of $f$. From a logical point of view, it is slightly curious that I now use 3.1 itself to construct extensions such as $f_{\Delta}$. The argument is made possible by 1.8, which furnishes extensions in special cases. The construction is by an infinite induction; it assumes $\mathcal{F}$ is locally defined, and $E$ is to be Lindelöf ([7],3.8), so that, at each stage, only finitely many sets, all convex, need be considered. "Countable" arguments of a similar kind can be carried out for some purposes in spaces that are merely paracompact, by virtue of the theorem of Stone that open coverings have $\sigma$-discrete refinements ([7], 5.1.12), but such refinements must be obtained by modifying the geometrical shape of the original sets of the cover. One cannot assume here that the sets of the refinement would still be convex. Moreover, at each stage one may also have to deal with infinitely many "predecessors", namely the individual sets of the preceding discrete classes.

For remarks about the details of the proof below, see 4.3.

REMARK 4.1. Let $\mathcal{U}, \mathcal{V}$ be open coverings of a topological space $S$. Then $\mathcal{V}$ is a strong star-refinement of $\mathcal{U}$ if, whenever $U \in \mathcal{V}$, there exists some member $T$ of $\mathcal{U}$ such that, whenever $V, W \in \mathcal{V}$ and $U \cap V \neq \emptyset \neq V \cap W$, necessarily $U \cup V \cup W \subseteq T$. 
This is not quite the same as a "star-refinement" as usually defined (see [7], 5.1.11, p. 376, and the discussion afterwards), but it remains true that any open covering of a paracompact space has a strong star-refinement; indeed, it suffices to take a star-refinement of a star-refinement.

Proposition 4.2. Let $E$ be a Hausdorff locally convex Lindelöf space, and $F$ a vector space, with $X$ a subset of $E$ and $Y$ a convex subset of $F$. Let $\mathcal{F}$ be a $Y$-admissible (see 1.7) and locally defined (see 1.4) class of functions on the open subsets of $X$ in the subspace topology, with values in $Y$. Suppose that $\mathcal{A}:=\left\{A_{u}: 1 \leq u \leq v\right\}$ is a complex consisting of open convex sets of $E$, and $\mathcal{B}$ is a complex of subsets of $E$, such that $\mathcal{B} \prec \mathcal{A}$ and $\bigcap_{u=1}^{v} A_{u} \neq \emptyset$. Write $A:=\bigcup_{u=1}^{v} A_{u}$ and $B:=\bigcup_{u=1}^{v} B_{u}$ (the carriers of $\mathcal{A}$ and $\left.\mathcal{B}\right)$. If $a$ function $f: A \cap X \rightarrow Y$ is $\mathcal{F}$-extensible (see 1.5), then there exists a function $g: X \rightarrow Y$ in $\mathcal{F}$ such that $g|B \cap X=f| B \cap X$.

Proof. (a) Since $\mathcal{F}$ itself may if necessary be translated, there is no loss of generality in supposing $0 \in \bigcap_{u=1}^{v} A_{u}$. Thus $N:=\bigcap_{u=1}^{v} A_{u}$ is a convex open neighbourhood of 0 in $E$.

As $\mathcal{B} \prec \mathcal{A}$, there is a convex neighbourhood $V$ of 0 in $E$ such that $B_{u}+V \subseteq A_{u}$ for each $u$, and, consequently,

$$
\left(B_{u} \cup\{0\}\right)+(V \cap N) \subseteq A_{u}, \quad \Gamma\left(B_{u} \cup\{0\}\right)+(V \cap N) \subseteq A_{u} .
$$

Set

$$
B_{u}^{\prime \prime}:=\Gamma\left(B_{u} \cup\{0\}\right)+\frac{1}{2}(V \cap N)
$$

( " $\Gamma$ " denotes the convex cover); this is a convex open neighbourhood of 0 in $E$. Let $B_{u}^{\prime}:=\operatorname{cl}_{E}\left(B_{u}^{\prime \prime}\right) \subseteq B_{u}^{\prime \prime}+\frac{1}{6}(V \cap N), B_{u}^{*}:=B_{u}^{\prime}+\frac{1}{6}(V \cap N)$; thus

$$
B_{u}^{*}+\frac{1}{6}(V \cap N) \subseteq A_{u} \cap\left(2 B_{u}^{\prime \prime}\right) .
$$

By taking $A_{u} \cap\left(2 B_{u}^{\prime \prime}\right)$ instead of $A_{u}, B_{u}^{\prime}$ instead of $B_{u}$, and $V$ instead of $\frac{1}{6}(V \cap N)$, I may therefore assume that, for each $u$,

$$
\left\{\begin{array}{l}
B_{u} \text { is a closed convex neighbourhood of } 0 ; \\
B_{u}^{*} \text { is another, such that } B_{u}+V \subseteq B_{u}^{*}, B_{u}^{*}+V \subseteq A_{u} \\
A_{u} \subseteq 2\left(\operatorname{int}_{E}\left(B_{u}\right)\right) .
\end{array}\right.
$$

The sets $A_{u}$ and $B_{u}$, and the unions $A:=\bigcup_{u=1}^{v} A_{u}$ and $B:=\bigcup_{u=1}^{v} B_{u}$, are then all star-shaped about 0 (a fact constantly employed below), and $\frac{1}{2} A \subseteq \operatorname{int}_{E}(B)$.

(b) There are two classes of points in $E$. The first class, which I shall call $\mathfrak{X}$, consists of those points $x$ (including 0 ) such that, for all $t \in(0, \infty)$, $t x \in A$. The second, $\mathfrak{Y}$, consists of those $y \in E \backslash\{0\}$ for which $t y \notin A$ for some $t \in(0, \infty)$.

Since $A$ is a finite union of the sets $A_{u}, \mathfrak{X}=\bigcup_{u=1}^{v} \mathfrak{X}_{u}$, where $\mathfrak{X}_{u}$ consists of the elements $x \in \mathfrak{X}$ such that $(0, \infty) x \subseteq A_{u}$. (This is not a disjoint union.) 
If $y \notin \mathfrak{X}_{u}$, there is some $t>0$ such that $t y \notin A_{u}$; then $(t+1) y \notin \operatorname{cl}_{E}\left(A_{u}\right)$, and $(t+1) z \notin \operatorname{cl}_{E}\left(A_{u}\right)$ for all $z$ in a suitably small neighbourhood of $y$. Thus $\mathfrak{X}_{u}$ is closed in $E$. (It is a convex cone, since $A_{u}$ is convex, and, by (14), one also has $(0, \infty) x \subseteq B_{u}$ for any $\left.x \in \mathfrak{X}_{u}\right)$. Hence $\mathfrak{Y}$, the complement of the union of the $\mathfrak{X}_{u}$, is open in $E$.

(c) If $y \in \mathfrak{Y}$ and $1 \leq u \leq v$, there exists $\lambda_{u}(y)>0$ such that $\lambda_{u}(y) y$ is in the frontier of $B_{u}^{*}$ in $E$; namely, $\lambda_{u}(y)=\left(p_{u}(y)\right)^{-1}$, where $p_{u}$ is the Minkowski functional of $\operatorname{int}_{E}\left(B_{u}^{*}\right)$, which takes positive values on $\mathfrak{Y}$ by definition. The function $\lambda_{u}$ is continuous on $\mathfrak{Y}$, since $p_{u}$ is.

If $\partial B^{*}$ denotes the frontier of $B^{*}$ in $E$, then $\partial B^{*} \subseteq \mathfrak{Y}$. In particular, $\mathfrak{Y}=(0, \infty) \partial B^{*} ;$ to any $y \in \mathfrak{Y}$ there corresponds a unique point $\Pi(y):=$ $\lambda(y) y$ of $\partial B^{*}$, where $\lambda(y) \in(0, \infty)$. It is clear that $\lambda(y)=\max _{1 \leq u \leq v} \lambda_{u}(y)$, so that $\lambda$ is a continuous positive-valued function on $\mathfrak{Y}$. Hence

$$
\left\{\begin{array}{l}
\text { the mapping }(0, \infty) \times \partial B^{*} \rightarrow \mathfrak{Y}:(\alpha, x) \mapsto \alpha x \\
\text { is a homeomorphism when } \partial B^{*} \text { is given the subspace topology; } \\
\text { its inverse is } y \mapsto\left(\lambda(y)^{-1}, \lambda(y) y\right) .
\end{array}\right.
$$

(d) For each $y \in \partial B^{*}$, the segment $\left[\frac{1}{2} y, y\right]$ lies in $A \cap \mathfrak{Y}$. There exists some $\varepsilon_{y} \in\left(0, \frac{1}{6}\right)$ such that $\left[\left(\frac{1}{2}-\varepsilon_{y}\right) y,\left(1+\varepsilon_{y} g\right) y\right] \subseteq A$. Apply 1.8: there are a convex open neighbourhood $W_{y}$ of 0 in $E$ and a function $k_{y}: X \rightarrow Y$ in $\mathcal{F}$ such that

$$
\left\{\begin{array}{l}
{\left[\left(\frac{1}{2}-\varepsilon_{y}\right) y,\left(1+\varepsilon_{y}\right) y\right]+2 W_{y} \subseteq A} \\
k_{y} \mid\left(\left[\left(\frac{1}{2}-\varepsilon_{y}\right) y,\left(1+\varepsilon_{y}\right) y\right]+W_{y}\right) \cap B^{*} \cap X \\
\quad=f \mid\left(\left[\left(\frac{1}{2}-\varepsilon_{y}\right) y,\left(1+\varepsilon_{y}\right) y\right]+W_{y}\right) \cap B^{*} \cap X .
\end{array}\right.
$$

I may also suppose that $\left(y+2 W_{y}\right) \cap B=\emptyset$, which necessarily implies that

$$
\left([1, \infty)\left(y+2 W_{y}\right)\right) \cap B=\emptyset .
$$

By (15), and by compactness in the product space, there exists a relatively open set $W_{y}^{*}$ in $\partial B^{*}$ which satisfies

$$
\left\{\begin{array}{l}
W_{y}^{*} \subseteq y+W_{y} \\
\left(\frac{1}{2}-\varepsilon_{y}, 1+\varepsilon_{y}\right) W_{y}^{*} \subseteq\left[\left(\frac{1}{2}-\varepsilon_{y}\right) y,\left(1+\varepsilon_{y}\right) y\right]+W_{y}
\end{array}\right.
$$

(the latter is open in $\mathfrak{Y}$ ). The sets $W_{y}$ and $W_{y}^{*}$ are to be chosen for each $y \in \partial B^{*}$.

Now $\partial B^{*}$ is closed in $E$, which is Lindelöf; thus $\partial B^{*}$ is Lindelöf, and so paracompact. The relatively open covering $\left\{W_{y}^{*}: y \in \partial B^{*}\right\}$ of $\partial B^{*}$ has a relatively open strong star-refinement $\left\{W_{\gamma}^{\prime \prime}: \gamma \in \Gamma\right\}$ (see 4.1 above).

Given $z \in \partial B^{*}$, take $\gamma \in \Gamma$ such that $z \in W_{\gamma}^{\prime \prime}$. By construction, $W_{\gamma}^{\prime \prime}$ is included in some $W_{y}^{*}$. By (15), $\left(\frac{1}{2}-\varepsilon_{y}, 1+\varepsilon_{y}\right) W_{\gamma}^{\prime \prime}$ is an open neighbourhood of $z$ in $\mathfrak{Y}$. So there is an open convex neighbourhood $N_{z}$ of 0 in $E$ such that 
$3 N_{z} \subseteq B$ and

$$
\left[\frac{1}{2}, 1\right]\left(z+3 N_{z}\right) \subseteq\left(\frac{1}{2}-\varepsilon_{y}, 1+\varepsilon_{y}\right) W_{\gamma}^{\prime \prime}
$$

(notice that $\left[\frac{1}{2}, 1\right]\left(z+3 N_{z}\right)$ is open in $\left.E\right)$. Next, set

$$
D(z):=\left[\frac{1}{2}, \infty\right)\left(z+N_{z}\right), \quad D^{*}(z):=\left[\frac{1}{2}, \infty\right)\left(z+3 N_{z}\right) .
$$

Notice that $\mathrm{cl}_{E}(D(z)) \subseteq D(z)+N_{z} \subseteq D^{*}(z)$.

Finally, as $\partial B^{*}$ is Lindelöf, its open cover $\left\{\left(z+N_{z}\right) \cap \partial B^{*}: z \in \partial B^{*}\right\}$ has a countable subcover $\left\{z_{n}+N_{z_{n}}: n=1,2, \ldots\right\}$. Then $\left\{D\left(z_{n}\right): n \in \mathbb{N}\right\}$ is an open cover of $\left[\frac{1}{2}, \infty\right) \partial B^{*}$ by convex sets, and

$$
D_{n}:=D\left(z_{n}\right) \prec D_{n}^{*}:=D^{*}\left(z_{n}\right) \quad \text { for each } n \geq 1 \text {. }
$$

If $i, j, k \in \mathbb{N}$ and $D_{i}^{*} \cap D_{j}^{*} \neq \emptyset \neq D_{j}^{*} \cap D_{k}^{*}$, the construction above gives corresponding $\gamma_{i}, \gamma_{j}, \gamma_{k} \in \Gamma$ such that $W_{\gamma_{i}}^{\prime \prime} \cap W_{\gamma_{j}}^{\prime \prime} \neq \emptyset \neq W_{\gamma_{j}}^{\prime \prime} \cap W_{\gamma_{k}}^{\prime \prime}$; the radial projection on $\partial B^{*}$ of a common point of the $D^{*} \mathrm{~s}$ is a common point of the $W^{\prime \prime}$ s. Then, by construction, there is some $y(i) \in \partial B^{*}$ with $W_{\gamma_{i}}^{\prime \prime} \cup W_{\gamma_{j}}^{\prime \prime} \cup W_{\gamma_{k}}^{\prime \prime} \subseteq W_{y(i)}^{*}$. Let

$$
Z_{i}:=\left(\frac{1}{2}-\varepsilon_{y(i)}, \infty\right)\left(y(i)+W_{y(i)}\right)
$$

then, by (18) and (19),

$$
D_{i}^{*} \cup D_{j}^{*} \cup D_{k}^{*} \subseteq Z_{i} .
$$

(e) For each $n \geq 1$ and each $u$ with $1 \leq u \leq v,[1, \infty)\left(y(n)+2 W_{y(n)}\right)$ and $B_{u}$ are open, convex, and disjoint by (17). Thus there are a continuous linear functional $\lambda_{n u}$ and a positive number $\varepsilon_{n u}$ such that

$$
\lambda_{n u}(x) \begin{cases}>1 & \text { when } x \in y(n)+W_{y(n)}, \\ <1-\varepsilon_{n u} & \text { when } x \in B_{u} .\end{cases}
$$

If $x \in Z_{n}$ and $\lambda_{n u}(x) \leq 1$, then $x \in\left(\frac{1}{2}-\varepsilon_{y(n)}, 1\right)\left(y(n)+W_{y(n)}\right) \subseteq A$ (see (22) and (16)).

(f) Consider now, for $n=0,1, \ldots$, the three complexes

$$
\mathcal{D}(n):=\left\{D_{1}, \ldots, D_{n}\right\}, \quad \mathcal{D}^{*}(n):=\left\{D_{1}^{*}, \ldots, D_{n}^{*}\right\}, \quad \mathcal{Z}(n):=\left\{Z_{1}, \ldots, Z_{n}\right\} .
$$

For $n=0$, these complexes are all empty.

Suppose that, for each simplex $\Delta$ of $\mathcal{D}^{*}(n)$, a function $g_{n}(\Delta): X \rightarrow Y$ in $\mathcal{F}$ is constructed in such a way that the following two conditions are satisfied. If $\Delta=\emptyset$, and in particular when $n=0, g_{n}(\Delta)$ may be arbitrary (by $1.7, \mathcal{F} \neq \emptyset$ ).

(1) For any simplices $\Delta_{1}, \Delta_{2}$ of $\mathcal{D}^{*}(n)$, and any $i \in \Delta_{1} \cap \Delta_{2}$,

$$
g_{n}\left(\Delta_{1}\right)\left|D_{i} \cap X=g_{n}\left(\Delta_{2}\right)\right| D_{i} \cap X .
$$

As a consequence, the mappings $g_{n}(\Delta)$ for the various simplices $\Delta$ of $\mathcal{D}^{*}(n)$ fit together to define a mapping $g_{n}: D(n) \cap X \rightarrow Y$ in $\mathcal{F}$, although they may not agree elsewhere. Recall from 2.10 that $D(n):=\bigcup_{i=1}^{n} D_{i}$. 
(2) For $1 \leq i \leq n$,

$$
\left\{\begin{array}{l}
g_{n}\left|D_{i} \cap R_{n} \cap X=f\right| D_{i} \cap R_{n} \cap X, \quad \text { where } \\
R_{n}:=\bigcap_{j=1}^{n}\left\{y \in E:(\exists k)\left(1 \leq k \leq v \& \lambda_{j k}(y) \leq 1-\frac{n}{n+1} \varepsilon_{j k}\right)\right\} .
\end{array}\right.
$$

$R_{n}$ is closed in $E$, and a moment's thought shows (with (e)) that

$$
R_{n+1} \subseteq \operatorname{int}_{E}\left(R_{n}\right), \quad Z_{n} \cap R_{n} \subseteq B^{*}, \quad B \subseteq R_{n} .
$$

The aim now, given the inductive assumptions (f)(1) and (2), is to construct $g_{n+1}(\Delta)$ for each simplex $\Delta$ of $\mathcal{D}^{*}(n+1)$. The induction may start when $n=0$; then $\mathcal{D}^{*}(0)=\emptyset$ and $D^{*}(0)=\emptyset$, and the argument below still works (rather trivially).

(g) Suppose first that $\Delta$ is a maximal simplex of $\mathcal{D}^{*}(n+1)$ such that $D^{*}(n+1)^{\Delta} \cap D_{n+1}^{*}=\emptyset$. Then $\Delta$ must be a (maximal) simplex of $\mathcal{D}^{*}(n)$. Thus it makes sense to let $g_{n+1}(\Delta):=g_{n}(\Delta)$.

(h) For convenience of notation, temporarily rearrange the indices $\{1, \ldots, n+1\}$ so that all the vertices of the simplices $\Delta$ of $\mathcal{D}^{*}(n+1)$ for which $D^{*}(n+1)^{\Delta} \cap D_{n+1}^{*} \neq \emptyset$ are listed as $\{p+1, p+2, \ldots, n+1\}$, with $q+1, q+2, \ldots, n+1$ all the indices $j$ for which $D_{j}^{*} \cap D_{n+1}^{*} \neq \emptyset$. (Here $p$ and $q$ of course depend on $n$, and $q \geq p$; furthermore, if $q=n$, then necessarily $p=q$ ). Thus, whenever $p<i \leq n+1$, there are a maximal simplex $\Delta \ni i$ in $\mathcal{D}^{*}(n+1)$, and a vertex $j \in \Delta$, such that $q<j \leq n+1$ and $D_{i}^{*} \cap D_{j}^{*} \neq \emptyset \neq D_{j}^{*} \cap D_{n+1}^{*}$. By the construction in (d), then,

$$
D_{n+1}^{*} \subseteq Z_{i} \quad \text { and } \quad D_{i}^{*} \subseteq Z_{n+1} \quad \text { for } p \leq i \leq n+1 .
$$

(However, remember that the labels $i$ and $n+1$ are only temporary.)

(i) Introduce the temporary notations $\mathcal{D}_{n, p}^{*}:=\left\{D_{p+1}^{*}, \ldots, D_{n}^{*}\right\}$ and $\mathcal{D}_{n, p}:=\left\{D_{p+1}, \ldots, D_{n}\right\}$. These complexes may be empty, if $p=n$. Certainly, $\mathcal{D}_{n, p} \prec \mathcal{D}_{n, p}^{*}$, as was pointed out at (21). Any simplex $\Delta$ of $\mathcal{D}_{n, p}^{*}$ is a simplex of $\mathcal{D}^{*}(n)$, so $g_{n}(\Delta): X \rightarrow Y$ is defined. The conditions of 3.1 are satisfied if I choose " $\mathcal{A}$ " to be $\mathcal{D}_{n, p}^{*}$ and " $\mathcal{B}$ " to be $\mathcal{D}_{n, p}$, and let the function $g_{n}: D_{n, p} \cap X \rightarrow Y$ (whose existence was assured by the inductive hypothesis (f)(1); here $D_{n, p}:=D_{p+1} \cup \ldots \cup D_{n}$ ) take the place of the " $f$ " of 3.1. From 3.1, then, there exists a function, which I call $h_{n}: X \rightarrow Y$, in $\mathcal{F}$, such that

$$
h_{n}\left|D_{n, p} \cap X=g_{n}\right| D_{n, p} \cap X .
$$

However, I have no control over the behaviour of $h_{n}$ near the origin (see (20)).

In addition, $Z_{n+1}$ was defined (see (16), (22), and (25)) so that there is a function $k_{n+1}: X \rightarrow Y$ in $\mathcal{F}$ (previously called $k_{y(n+1)}$ ) for which

$$
k_{n+1}\left|Z_{n+1} \cap B^{*} \cap X=f\right| Z_{n+1} \cap B^{*} \cap X .
$$


(j) Let $\Lambda_{n}: E \rightarrow\left(\mathbb{R}^{v}\right)^{n}: x \mapsto\left(\lambda_{j 1}(x), \ldots, \lambda_{j v}(x)\right)_{j=1}^{n}$. Represent $\left(\mathbb{R}^{v}\right)^{n}$ as the space of $n \times v$ matrices $M_{n v}$, denoting by $e_{j i}: M_{n v} \rightarrow \mathbb{R}$ the map which selects the $(j, i)$ th entry.

Since the functionals $\lambda_{j i}$ may not be linearly independent, $\Lambda_{n}$ need not be surjective; call its image $T_{n} \subseteq M_{n v}$, a finite-dimensional real vector space. Let

$$
\begin{aligned}
K_{n} & :=\left\{\xi \in T_{n}:(\forall j)(\exists k) 1 \leq k \leq v \& e_{j k}(\xi) \leq 1-\frac{n+1}{n+2} \varepsilon_{j k}\right\}, \\
J_{n} & :=\left\{\xi \in T_{n}:(\forall j)(\exists k) 1 \leq k \leq v \& e_{j k}(\xi)<1-\frac{n}{n+1} \varepsilon_{j k}\right\},
\end{aligned}
$$

so that $J_{n}, K_{n}$ are convex sets in $T_{n} ; K_{n} \prec J_{n}, K_{n}$ is closed and $J_{n}$ is open. Construct a $\mathrm{C}^{\infty}$ function $\psi_{n}: T_{n} \rightarrow[0,1]$ with $\psi_{n}\left(K_{n}\right)=\{1\}$ and $\psi_{n}\left(E \backslash J_{n}\right)=\{0\}$. So, $\psi_{n} \circ \Lambda_{n}$ takes the value 1 on $R_{n+1} \subseteq \Lambda_{n}^{-1}\left(K_{n}\right)$ (see (24)) and the value 0 on $E \backslash\left(\operatorname{int}_{E}\left(R_{n}\right)\right)$, since $\Lambda_{n}^{-1}\left(J_{n}\right) \subseteq \operatorname{int}_{E}\left(R_{n}\right)$. Now combine the functions $h_{n}$ and $k_{n}$ of (i) by defining, for $x \in X$,

$$
h_{n}^{\prime}(x):=\psi_{n}\left(\Lambda_{n}(x)\right) k_{n+1}(x)+\left(1-\psi_{n}\left(\Lambda_{n}(x)\right)\right) h_{n}(x) .
$$

As $\mathcal{F}$ is admissible, $h_{n}^{\prime}$ also belongs to $\mathcal{F}$.

(k) If $x \in D_{i} \cap X$ for some $i$ with $p<i \leq n$, there are two cases. If $x \notin \operatorname{int}_{E}\left(R_{n}\right)$, then $(29)$ gives $h_{n}^{\prime}(x)=h_{n}(x)=g_{n}(x)$, by (27) and (f)(1). If $x \in D_{i} \cap R_{n+1} \cap X$, then $g_{n}(x)=f(x)$ by the inductive hypothesis (f) (2) and therefore $h_{n}(x)=f(x)$ by (27). At the same time, from (23) and (25),

$$
D_{i} \cap R_{n+1} \subseteq Z_{i} \cap R_{i} \subseteq B^{*},
$$

so that $D_{i} \cap R_{n+1} \cap X \subseteq Z_{n+1} \cap B^{*} \cap X$ by (26). Consequently, by (28), $k_{n+1}(x)=f(x)$ as well - so $h_{n}^{\prime}(x)=g_{n}(x)$ once more. Hence

$$
\left\{\begin{array}{l}
h_{n}^{\prime}\left|D_{i} \cap R_{n+1} \cap X=f\right| D_{i} \cap R_{n+1} \cap X, \\
h_{n}^{\prime}\left|D_{i} \cap X=g_{n}\right| D_{i} \cap X \quad \text { for } p<i \leq n .
\end{array}\right.
$$

If $x \in Z_{n} \cap R_{n+1} \cap X$, then $h_{n}^{\prime}(x)=k_{n+1}(x)$, by (29). From (26) and (28),

$$
h_{n}^{\prime}\left|D_{n+1} \cap R_{n+1} \cap X=k_{n+1}\right| D_{n+1} \cap R_{n+1} \cap X .
$$

(l) If $D^{*}(n+1)^{\Delta} \cap D_{n+1}^{*} \neq \emptyset$, where $\Delta$ is a maximal simplex in the complex $\mathcal{D}^{*}(n+1)$, then let $g_{n+1}(\Delta):=h_{n}^{\prime}$, as just constructed in $(\mathrm{k})$. This, with $(\mathrm{g})$, defines $g_{n+1}(\Delta)$ for all maximal simplices of $\mathcal{D}^{*}(n+1)$.

(m) With each non-maximal simplex $\Delta$ of $\mathcal{D}^{*}(n+1)$, associate (once and for all) a maximal simplex $\Delta^{\prime} \supseteq \Delta$, and define $g_{n+1}(\Delta):=g_{n+1}\left(\Delta^{\prime}\right)$.

(n) If $\Delta_{1}, \Delta_{2}$ are simplices of $\mathcal{D}^{*}(n+1)$ and $i \in \Delta_{1} \cap \Delta_{2}$, then take the maximal simplices $\Delta_{1}^{\prime}, \Delta_{2}^{\prime}$ of $\mathcal{D}^{*}(n+1)$ that were fixed at $(\mathrm{m})$; certainly, $i \in \Delta_{1}^{\prime} \cap \Delta_{2}^{\prime}$. If the stars of both $\Delta_{1}^{\prime}$ and $\Delta_{2}^{\prime}$ meet $D_{n+1}^{*}$, as at (1), then 
$g_{n+1}\left(\Delta_{1}^{\prime}\right)$ and $g_{n+1}\left(\Delta_{2}^{\prime}\right)$ are both $h_{n}^{\prime}$, and automatically

$$
g_{n+1}\left(\Delta_{1}^{\prime}\right)\left|D_{i} \cap X=g_{n+1}\left(\Delta_{2}^{\prime}\right)\right| D_{i} \cap X .
$$

If $i \leq n$, (30) shows that both of them agree with $g_{n} \mid D_{i} \cap X$.

If only the star of $\Delta_{1}^{\prime}$ in $\mathcal{D}^{*}(n+1)$ meets $D_{n+1}^{*}$, then, again, $i \leq n$ and $\Delta_{2}^{\prime}$ is a simplex of $\mathcal{D}^{*}(n)$ and $g_{n+1}\left(\Delta_{2}^{\prime}\right)=g_{n}\left(\Delta_{2}^{\prime}\right)$ by (g); whilst, by (30),

$$
g_{n+1}\left(\Delta_{1}^{\prime}\right)\left|D_{i} \cap X=h_{n}^{\prime}\right| D_{i} \cap X=g_{n}\left|D_{i} \cap X=g_{n}\left(\Delta_{2}^{\prime}\right)\right| D_{i} \cap X .
$$

Lastly, if the star in $\mathcal{D}^{*}(n+1)$ of neither $\Delta_{1}^{\prime}$ nor $\Delta_{2}^{\prime}$ meets $D_{n+1}^{*}$, then, by $(\mathrm{g})$ and $(\mathrm{f})(1)$,

$$
g_{n+1}\left(\Delta_{1}^{\prime}\right)\left|D_{i} \cap X=g_{n}\left(\Delta_{1}^{\prime}\right)\right| D_{i} \cap X=g_{n}\left(\Delta_{2}^{\prime}\right)\left|D_{i} \cap X=g_{n+1}\left(\Delta_{2}^{\prime}\right)\right| D_{i} \cap X .
$$

Thus the inductive hypothesis (f)(1) still holds when $n$ is substituted by $n+1$, and also $g_{n+1}\left|D_{i} \cap X=g_{n}\right| D_{i} \cap X$ for $1 \leq i \leq n$, or

$$
g_{n+1}\left|D(n) \cap X=g_{n}\right| D(n) \cap X .
$$

(o) The hypothesis (f)(2) must also be considered. If $1 \leq i \leq p$, and $\Delta$ is a maximal simplex of $\mathcal{D}^{*}(n+1)$ with $i \in \Delta$, then (g) applies because of the definition of $p$ (see $(\mathrm{h})$ ). Thus

$$
g_{n+1}(\Delta)\left|D_{i} \cap R_{n+1} \cap X=g_{n}(\Delta)\right| D_{i} \cap R_{n+1} \cap X=f \mid D_{i} \cap R_{n+1} \cap X,
$$

by (f)(2)-recall from (24) that $R_{n+1} \subseteq R_{n}$. If $p<i \leq n+1$, choose a maximal simplex $\Delta \subseteq\{p+1, \ldots, n+1\}$ with $i \in \Delta$ and $D^{*}(n+1)^{\Delta} \cap D_{n+1}^{*}$ $\neq \emptyset$, which is possible, again by the definition of $p$. Hence, by $(\mathrm{k})$ and by (30), for $p<i \leq n$,

$$
g_{n+1}(\Delta)\left|D_{i} \cap R_{n+1} \cap X=h_{n}^{\prime}\right| D_{i} \cap R_{n+1} \cap X=f \mid D_{i} \cap R_{n+1} \cap X,
$$

whilst, for $i=n+1,(31)$, (26), and (28) yield

$$
g_{n+1}(\Delta)\left|D_{n+1} \cap R_{n+1} \cap X=k_{n+1}\right| D_{n+1} \cap R_{n+1} \cap X=f \mid D_{i} \cap R_{n+1} \cap X \text {. }
$$

In either case, the inductive hypothesis (f)(2) still holds when $n+1$ takes the place of $n$. Now restore the original order of the first $n+1$ indices. The inductive construction may be carried out for all $n$, giving a mapping $g_{n}(\Delta): X \rightarrow Y$ for each $n$ and each simplex $\Delta$ of $\mathcal{D}^{*}(n)$.

(p) Let $D(\infty):=\bigcup_{n=1}^{\infty} D_{n}$. By the definition of the sets $D_{n}$ in (d), $D(\infty) \supseteq\left[\frac{1}{2}, \infty\right) \partial B^{*}$. If $x \in E \backslash D(\infty)$, then either $x \in\left(0, \frac{1}{2}\right) \partial B^{*}$ or $(0, \infty) x \subseteq B^{*}$. Since $B^{*} \subseteq A$ and $\frac{1}{2} A \subseteq B$ (see (14)), both these possibilities give $x \in \operatorname{int} B$; hence $D(\infty) \cup(\operatorname{int} B)=E$. A mapping

$$
g(\infty): D(\infty) \cap X \rightarrow Y
$$

may be defined by setting $g(x):=g_{n}(x)$, where $n$ is the first index such that $x \in D(n)$. Because of (32), any larger index could also be taken, so that $g(\infty)\left|D_{p}=g_{p}\right| D_{p}$ for any $p$; as $g_{p} \in \mathcal{F}, D_{p}$ is open, and $\mathcal{F}$ is locally defined, it follows that $g(\infty)$ is in $\mathcal{F}$. 
However, by (f)(2) (with (25)), $g(\infty)\left|D_{n} \cap B \cap X=f\right| D_{n} \cap B \cap X$ for all $n$. Thus $g(\infty)$ agrees with $f$ on $D(\infty) \cap B \cap X$, and we may define $g: X \rightarrow Y$ by

$$
g|D(\infty) \cap X=g(\infty), \quad g|(\operatorname{int} B) \cap X=f \mid(\operatorname{int} B) \cap X .
$$

This function $g$ is in $\mathcal{F}$, and has the required properties.

REMARK 4.3. The details of the proof could be varied in many ways. The technical difficulty of the argument here is the necessity of obtaining strong star-refinements by radial projection on $\partial B^{*}$, which is awkward because the $D_{n}$ have to be convex in $E$ (to allow for the introduction of linear functionals) as well as refining the cones on the $W_{\gamma}^{\prime \prime}$.

The introduction of the sets $R_{n}$ is needed to "correct" the mapping $k_{n}$ given by 3.1, and ensure it agrees with $f$ on $B$. It appears impossible to arrange that $g_{n}(\Delta)\left|D^{*}(n)=g_{n+1}(\Delta)\right| D^{*}(n)$ for all the simplices $\Delta$ of $\mathcal{D}^{*}(n)$; the reason is that the application of 3.1 preserves the restriction of the function to $D(n)^{\Delta}$, but may change it on the larger set $D^{*}(n)^{\Delta}$.

In 3.1, the hypothesis that $\mathcal{B} \prec \mathcal{A}$ was important for (8). In 4.2 , which is perhaps only interesting in conjunction with 3.1 , it is natural and convenient, but not really necessary. The only point at which it is used seems to be the construction in (a) of the "intermediate" closed convex sets $B_{u}^{*}$ such that $B_{u} \subseteq \operatorname{int}_{E} B_{u}^{*}$ and $B_{u}^{*} \subseteq A_{u}$. If $E$ is a normed space, 2.3 shows that this construction is possible as soon as $B_{u}$ is a closed convex subset of $A_{u}$. I do not know how this result can be extended to more general locally convex spaces. However, if $B_{u}$ has non-empty interior, 2.7 constructs $B_{u}^{*}$.

On the other hand, the proof of 4.2 does not, in fact, require the convexity of $B_{u}^{*}$, but only the facts that it is star-shaped (about the origin, by convention) and that its frontier meets each radius in at most one point. The construction of a $B_{u}^{*}$ satisfying these weaker conditions can be carried out much more generally. The interest of such questions is that, given some specific class $\mathcal{F}$ on $E$, one would like to know for exactly which pairs $(U, C)$ of an open set $U$ and a closed subset $C$ in $E$ any function $f$ defined on $U$ and belonging to $\mathcal{F}$ corresponds to some function $g$ of class $\mathcal{F}$ on $E$ such that $g \mid C=f$. The remarks at 3.3 on other notions of convexity are relevant to this.

A rough summary of 4.2 and of 3.1 would be that a function which can be extended locally to $E$ (is "extensible") can also be extended globally from suitable sets. It is global extensibility which is required in practice.

DeFinition 4.4. Let $\mathcal{F}$ be an affine space of functions on the topological space $X$, with values in the convex subset $Y$ of the vector space $F$. Suppose $U$ is an open subset of $X$, and $\mathcal{E}$ is a convex set of functions $U \rightarrow F$. Then $\mathcal{E}$ is said to admit local affine $\mathcal{F}$-extension operators if, for every point 
$x \in U$, there is an open neighbourhood $V$ of $x$ in $U$ and an affine mapping $\mathcal{L}_{V}: \mathcal{E} \rightarrow \mathcal{F}(X ; Y)$ such that, for each $f \in \mathcal{E}, \mathcal{L}_{V}(f)|V=f| V$.

There is a similar definition for "linear extension operators".

Corollary 4.5. Let $\mathcal{A}, \mathcal{B}$ and $\mathcal{F}, E, F, X$ be as in 4.2 , and let $Y$ be a convex set in $F$. Let $\mathcal{F}$ be a $Y$-admissible and locally defined affine space of functions on the open sets of $X$, with images in $Y$. Suppose that $\mathcal{E}$ is a convex set of functions $A \cap X \rightarrow Y$ in $\mathcal{F}$ which admits local affine $\mathcal{F}$-extension operators. Then there is an affine-linear map $\mathcal{L}: \mathcal{E} \rightarrow \mathcal{F}(X ; Y)$ such that $\mathcal{L}(f)|B \cap X=f| B \cap X$ for each $f \in \mathcal{E}$.

Proof. Every step of the constructions in 1.8 and 4.2 is by a pointwise convex combination of the values of extensions either already constructed or given by the hypotheses. Where, at 4.2 (i), appeal was made to 3.1 , it should now be made to 3.4 .

There is of course a "linear" version, which is an easy corollary. Now recall 3.6.

Lemma 4.6. Suppose in 4.2 or in 4.5 that $E$ is a normed space and all the convex sets belonging to the complex $\mathcal{A}$ are bounded. Let $G$ be a normdense subspace of the dual space $E^{\prime}$. Then the previous conclusions in 4.2 or in 4.5 hold on the weaker condition that $\mathcal{F}$ is $(G, Y)$-admissible instead of $Y$-admissible.

Proof. The use of 3.1 or of 3.4 at 4.2 (i) should now be substituted by 3.7. The neighbourhoods $W_{y}$ of the origin may be norm-balls, which are bounded, so that, as was shown in 3.7, the linear functionals $\lambda_{n u}$ of $4.2(\mathrm{e})$ may be chosen from $G$.

The full dual space $E^{\prime}$ also appeared in the proof of 1.8 , when $\phi$ was chosen as a support functional for $W$ at $\kappa c$ to arrange (1). However, one might instead choose any $\phi \in G$ such that $\phi(\kappa c)=1$, and substitute $W^{\prime}:=$ $W \cap \phi^{-1}(-1,1)$ for $W$ throughout the rest of the proof. ( $W$ only had to be sufficiently small, convex and symmetric.)

\section{The main theorems}

THEOREM 5.1. Let E be a Hausdorff locally convex Lindelöf space, and $F$ a vector space, with $X$ a subset of $E$ and $Y$ a convex subset of $F$. Let $\mathcal{F}$ be a $Y$-admissible and locally defined class of functions on the open subsets of $X$, with values in $Y$. Suppose that $\mathcal{A}:=\left\{A_{1}, \ldots, A_{v}\right\}$ is a complex of open convex sets of $E$ and $\mathcal{B}:=\left\{B_{1}, \ldots, B_{v}\right\} \prec \mathcal{A}$. Let $A$ denote the carrier of $\mathcal{A}$ and $B$ the carrier of $\mathcal{B}$, and suppose that there are given a mapping $f: B \cap X \rightarrow Y$ and, for each simplex $\Delta$ of $\mathcal{A}$, an $\mathcal{F}$-extensible mapping $f_{\Delta}: A^{\Delta} \cap X \rightarrow Y$ such that $f_{\Delta}\left|B^{\Delta} \cap X=f\right| B^{\Delta} \cap X$. Then there exists a function $h: X \rightarrow Y$ in $\mathcal{F}$ such that $h|B \cap X=f| B \cap X$. 
Proof. For each simplex $\Delta$ of $\mathcal{A}, 4.2$ may be applied to the complexes

$$
\mathcal{A}^{\Delta}:=\left\{A_{u}: u \in \Delta\right\}, \quad \mathcal{B}^{\Delta}:=\left\{B_{u}: u \in \Delta\right\} ;
$$

so there exists $g_{\Delta}: X \rightarrow Y$ in $\mathcal{F}$ such that $g_{\Delta}\left|B^{\Delta} \cap X=f\right| B^{\Delta} \cap X$. Proposition 3.1 may be applied in its turn to give the result.

The statement above is convenient for later applications, because it does not require that the mappings $f_{\Delta}$ should fit together to define a mapping of the whole of $A \cap X$, but it is less striking than the following.

Corollary 5.2. Let $\mathcal{A}, \mathcal{B}$ and $\mathcal{F}, E, F, X, Y, A, B$ be as in 5.1 , and let $k: A \cap X \rightarrow Y$ be $\mathcal{F}$-extensible. Then there exists a function $q: X \rightarrow Y$ in $\mathcal{F}$ such that $q|B \cap X=k| B \cap X$.

Proof. In 5.1 take $f_{\Delta}:=k \mid A^{\Delta} \cap X$ for each simplex $\Delta$ of $\mathcal{A}$.

Theorem 5.3. Let $\mathcal{A}, \mathcal{B}$ and $\mathcal{F}, E, F, X, Y, A, B$ be as in 5.1. Let $\mathcal{F}$ be a $Y$-admissible and locally defined affine space of functions on the open sets of $X$, with images in $Y$. Suppose that $\mathcal{E}$ is a convex set of functions $A \cap X \rightarrow Y$ which admits local affine $\mathcal{F}$-extension operators. Then there is an affine-linear map $\mathcal{L}: \mathcal{E} \rightarrow \mathcal{F}(X ; Y)$ such that

$$
\mathcal{L}(f)|B \cap X=f| B \cap X
$$

for each $f \in \mathcal{E}$.

Proof. Collate 4.5 and 3.4, exactly as in the proof of 5.1 .

Of course 5.2 is the special case of 5.3 when $\mathcal{E}:=\{f\}$.

REMARK 5.4. In normed spaces, the results above may be improved; that is, one may weaken the conditions on $\mathcal{F}$ to require only $(G, Y)$-admissibility as defined at 3.6. This is probably a significant fact. At 3.7 and 4.6 , it depended on the boundedness of the sets of the complexes, but this restriction can now be removed. (It is not clear, however, that the further theory benefits substantially from this generalization).

A normed (or metric) space is Lindelöf if and only if it is separable.

THEOREM 5.5. Let $E$ be a separable normed space, and $F$ a vector space, with $X$ a subset of $E$ and $Y$ a convex subset of $F$. Let $G$ be a norm-dense subspace of the dual space $E^{\prime}$. Let $\mathcal{F}$ be a $(G, Y)$-admissible and locally defined class of functions on the open subsets of $X$, with values in $Y$. Suppose that $\mathcal{A}:=\left\{A_{1}, \ldots, A_{v}\right\}$ and $\mathcal{B}:=\left\{B_{1}, \ldots, B_{v}\right\}$ are complexes of subsets of $E$, where $\mathcal{A}$ consists of open convex sets of $E$ and $\mathcal{B} \preceq \mathcal{A}$ (see 2.4 and 2.9), and let $A$ denote the carrier of $\mathcal{A}$ and $B$ the carrier of $\mathcal{B}$. Let $\mathcal{E}$ be a convex set of functions $A \cap X \rightarrow Y$ in $\mathcal{F}$ which admits local affine $\mathcal{F}$-extension operators. Then there is an affine-linear map $\mathcal{L}: \mathcal{E} \rightarrow \mathcal{F}(X ; Y)$ such that $\mathcal{L}(f)|B \cap X=f| B \cap X$ for each $f \in \mathcal{E}$. 
Proof. By 2.6, construct a sequence $\left(\mathcal{B}^{n}\right)_{n=1}^{\infty}$ of complexes of open convex sets such that $\mathcal{B} \preceq \mathcal{B}^{n+1} \preceq \mathcal{B}^{n} \preceq \mathcal{A}$ for all $n$. Here $\mathcal{B}^{n}$ stands for $\left\{B_{1}^{n}, \ldots, B_{v}^{n}\right\}$. I shall write $B^{n}$ for the carrier of $\mathcal{B}^{n}$, and let $\mathcal{B}^{0}:=\mathcal{A}$. Define, for $n=0,1, \ldots$,

$$
\left\{\begin{array}{c}
\mathcal{C}_{n}:=\left\{B_{E}(0 ; n), B_{E}(0 ; n+2) \cap B_{1}^{n}, B_{E}(0 ; n+2) \cap B_{2}^{n}, \ldots\right. \\
\left.\ldots, B_{E}(0 ; n+2) \cap B_{v}^{n}\right\}, \\
\mathcal{D}_{n}:=\left\{B_{E}(0 ; n-1), B_{E}(0 ; n+1) \cap B_{1}^{n+1}, \ldots\right. \\
\left.\ldots, B_{E}(0 ; n+1) \cap B_{v}^{n+1}\right\},
\end{array}\right.
$$

where, of course, $B_{E}(0 ; \varrho)=\emptyset$ when $\varrho \leq 0$, and the index set of the complexes is understood to be $\{0,1, \ldots, v\}$. It is clear that $\mathcal{D}_{n} \prec \mathcal{C}_{n}$ for all $n$, and that all the sets in $\mathcal{C}_{n}$ are bounded. Let $C_{n}, D_{n}$ denote the carriers of $\mathcal{C}_{n}$ and of $\mathcal{D}_{n}$.

There is an inductive procedure, which may be described as follows. Suppose that $f \in \mathcal{E} \subseteq \mathcal{F}(A \cap X ; Y)$. Since $B_{E}(0 ; 0)=\emptyset$, we have $C_{0} \cap X \subseteq$ $A \cap X$, and there is a linear restriction operator $i_{0}$, where $i_{0}(f):=f \mid C_{0} \cap X$. Set $\mathcal{E}_{0}:=i_{0}(\mathcal{E})$. Certainly, $\mathcal{E}_{0}$ admits local affine $\mathcal{F}$-extension operators, and, moreover, $f_{0}:=i_{0}(f)$ agrees with $f$ on $D_{0} \cap X=B_{E}(0 ; 1) \cap B^{1} \cap X$. Use 4.6 to construct a function $f_{1} \in \mathcal{F}(X ; Y)$ which depends linearly on $f_{0}$, and therefore on $f$, and agrees with $f$ on $D_{0} \cap X$.

Inductively, suppose for $n \geq 1$ that $f_{n} \in \mathcal{F}(X ; Y)$ is given and depends linearly on $f$; and that $f_{n}$ agrees with $f$ on $B_{E}(0 ; n+1) \cap B^{n+1} \cap X$. We may then define $g_{n} \in \mathcal{F}\left(C_{n+1} \cap X ; Y\right)$ by

$$
\left\{\begin{array}{l}
g_{n}\left|B_{E}(0 ; n+3) \cap B^{n+1} \cap X=f\right| B_{E}(0 ; n+3) \cap B^{n+1} \cap X, \\
g_{n}\left|B_{E}(0 ; n+1) \cap X=f_{n}\right| B_{E}(0 ; n+1) \cap X .
\end{array}\right.
$$

As $\mathcal{F}$ is locally defined, the function thus defined is indeed in $\mathcal{F}$, and it admits local affine $\mathcal{F}$-extension operators, as each of the restrictions does.

Since $\mathcal{D}_{n+1} \prec \mathcal{C}_{n+1}, 4.6$ now constructs a function $f_{n+1} \in \mathcal{F}(X ; Y)$ that agrees with $g_{n}$ on $D_{n+1} \cap X$. (I recall that this is for $n \geq 1$.) Furthermore, $f_{n+1}$ depends linearly on $f$. This completes the inductive construction.

As $f_{n+1}\left|D_{n+1} \cap X=g_{n}\right| D_{n+1} \cap X,(33)$ and (34) show that

$$
f_{n+1}\left|B_{E}(0 ; n) \cap X=f_{n}\right| B_{E}(0 ; n) \cap X
$$

for all $n \geq 1$. One may therefore define a function $g: X \rightarrow Y$ by the prescription

$$
g(x):=f_{n}(x) \quad \text { when } x \in B_{E}(0 ; n) \cap X .
$$

Since $\mathcal{F}$ is locally defined, $g \in \mathcal{F}(X ; Y)$, and it depends linearly on $f$; we may write $\mathcal{L}(f):=g$. However, look at the "other part" of $D_{n+1}$ in (33) and (34): 


$$
\begin{aligned}
f_{n+1} \mid B_{E}(0 ; n+2) \cap B^{n+2} \cap X & =g_{n} \mid B_{E}(0 ; n+2) \cap B^{n+2} \cap X \\
& =f \mid B_{E}(0 ; n+2) \cap B^{n+2} \cap X
\end{aligned}
$$

for all $n \geq 1$. Hence, from definition (35) (recall $B \subseteq B^{n+2}$ for all $n$ ),

$$
\begin{aligned}
g \mid B_{E}(0 ; n+1) \cap B \cap X & =f_{n+1} \mid B_{E}(0 ; n+1) \cap B \cap X \\
& =f \mid B_{E}(0 ; n+1) \cap B \cap X,
\end{aligned}
$$

which implies that $g|B \cap X=f| B \cap X$ as required.

ConCLUSION 5.6. In the sequel, I shall show how the above results may be extended to manifolds. The methods to be used are not unlike the proof of 4.2 ; that is, they depend on an induction of a similar type. It is worth noting that the proof of 5.5 already involves three inductions, none of them altogether trivial.

\section{References}

[1] R. A. Bonic and J. Frampton, Smooth functions on Banach manifolds, J. Math. Mech. 15 (1966), 877-898.

[2] R. Deville, A characterization of $C^{\infty}$-smooth Banach spaces, Bull. London Math. Soc. 22 (1990), 13-17.

[3] R. Deville, G. Godefroy and V. Zizler, Smoothness and Renormings in Banach Spaces, Pitman Monogr. Surveys Pure Appl. Math. 64, Longman, New York, 1992.

[4] J. Eells and K. D. Elworthy, Wiener integration on certain manifolds, in: Problems in Non-Linear Analysis (C.I.M.E., IV Ciclo, Varenna, 1970), Edizioni Cremonese, Roma, 1971, 67-94.

[5] K. D. Elworthy, Gaussian measures on Banach spaces and manifolds, in: Global Analysis and Its Applications (Trieste, 1972), International Atomic Energy Agency, Vienna, 1974, Vol. II, 151-166.

[6] - Embeddings, isotopy and stability of Banach manifolds, Compositio Math. 24 (1972), 175-226.

[7] R. Engelking, General Topology, PWN, Warszawa, 1977.

[8] H. Toruńczyk, Smooth partitions of unity in some non-separable Banach spaces, Studia Math. 46 (1973), 43-51.

[9] H. Triebel, Theory of Function Spaces, Monogr. Math. 78, Birkhäuser, BaselBoston, 1983.

[10] - , Theory of Function Spaces II, Monogr. Math. 84, Birkhäuser, Basel-Boston, 1992.

School of Mathematical and Computing Sciences

Victoria University of Wellington

P.O. Box 600

Wellington, New Zealand

E-mail: Chris.Atkin@vuw.ac.nz

Received March 16, 1999

Revised version January 16, 2001 